\title{
CSI-Microbes: Identifying cell-type specific intracellular microbes from single-cell RNA-seq data
}

Welles Robinson ${ }^{1,2,3^{*}}$, Fiorella Schischlik ${ }^{1}$, E. Michael Gertz ${ }^{1}$, Joo Sang Lee ${ }^{4}$, Kaiyuan Zhu ${ }^{1,5}$, S. Cenk Sahinalp ${ }^{1}$, Rob Patro ${ }^{2,3}$, Mark D.M. Leiserson ${ }^{2,3}$, Alejandro A. Schäffer ${ }^{\wedge}$, Eytan Ruppin $^{1 * *}$

${ }^{1}$ Cancer Data Science Laboratory, Center for Cancer Research, National Cancer Institute, Bethesda, MD, USA 20892

${ }^{2}$ Center for Bioinformatics and Computational Biology, University of Maryland, College Park, MD, USA 20910

${ }^{3}$ Department of Computer Science, University of Maryland, College Park, MD, USA 20910

${ }^{4}$ Department of Artificial Intelligence \& Department of Precision Medicine, School of Medicine, Sungkyunkwan University, Suwon 16419, Republic of Korea

${ }^{5}$ Department of Computer Science, Indiana University, Bloomington, IN, USA 47408

$\wedge$ Equal contributing last authors

* Corresponding authors

Address for correspondence:

Dr. Eytan Ruppin

National Cancer Institute, National Institutes of Health

Building 15-C1

Bethesda, MD 20892

Eytan.Ruppin@nih.gov 


\begin{abstract}
Despite recent reports of microbes living within tumor cells, the identification of intracellular microbes remains an open and important challenge. Here we introduce CSI-Microbes (computational identification of Cell type Specific Intracellular Microbes), a computational approach for the discovery of cell-type specific intracellular microbial taxa from single-cell RNA sequencing of host cells. It is first validated on two gold-standard datasets of human immune cells exposed to Salmonella. Next, CSI-Microbes is tested on Merkel cell and colorectal carcinoma where it identifies both reported and previously unknown tumor-specific intracellular microbes. Finally, CSI-Microbes is applied to analyze the intracellular microbiome of thirteen lung tumors where it identifies four tumors with bacterial taxa enriched in tumor cells and two tumors with bacterial taxa enriched in stroma or immune cells. Notably, the infected tumor cells down-regulate pathways associated with anti-microbial response and antigen processing and presentation, testifying to the functional significance of bacterial presence.
\end{abstract}




\section{Introduction}

Several recent papers have pointed to the functional importance of the tumor microbiome. For example, bacteria of the genus Fusobacterium are enriched in colorectal carcinoma compared to matched normal tissue, drive tumorigenesis, influence response to chemotherapy and bind to multiple human immune inhibitory receptors ${ }^{1-6} \cdot p k s+E$. coli have been shown to induce a mutation signature frequently found in colorectal carcinoma ${ }^{7}$. In pancreatic cancer, a subset of taxa from the class Gammaproteobacteria were shown to mediate tumor resistance to chemotherapy ${ }^{8}$. A computational analysis of the unmapped reads from whole-genome sequencing (WGS) and whole-transcriptome sequencing (RNA-seq) experiments across 33 tumor types from The Cancer Genome Atlas (TCGA) cohort identified a variety of bacterial genera present in different tumor types and demonstrated that after filtering out potentially contaminant species, one can successfully build a predictor of cancer type based on tumors' microbial composition ${ }^{9}$.

Some residents of the tumor microbiome have been shown to reside intracellularly within tumor and non-tumor cells in the tumor microenvironment ${ }^{1,10}$. The previously mentioned Fusobacterium has been shown to bind to ligands overexpressed at the surface of colorectal carcinoma cells, which it uses to invade these cells ${ }^{1,11}$. Another recent publication used multiple experimental techniques to interrogate the microbiome of seven cancer types ${ }^{10}$. It found that each cancer type has its own characteristic tumor microbiome and that many intratumoral bacteria exist intracellularly in both tumor and immune cells ${ }^{10}$. Further, it was recently reported that peptides derived from proteins in 41 bacterial species, including Fusobacterium nucleatum, are presented on the human leukocyte antigen class I and II (HLA-I and HLA-II) molecules of melanoma cells, which suggests that intracellular bacteria antigens can possibly be exploited 
therapeutically ${ }^{12}$. Despite these advances, it has remained an important open challenge to identify which microbial taxa reside intracellularly and whether they reside exclusively or preferentially in tumor cells, immune cells, or cells of the non-cancerous solid tissue adjacent to the solid tumor (e.g., the study that identified the intracellular localization of some bacteria using staining also characterized the composition of the tumor microbiomes using $16 \mathrm{~S}$ ribosomal $\mathrm{RNA}^{13,14}$ but did not attempt to classify which bacterial taxa resided intracellularly in which cell types).

Here, we present a computational approach named CSI-Microbes (computational identification of Cell-type Specific Intracellular Microbes) that analyzes single cell RNA sequencing (scRNA-seq) datasets to identify intracellular microbes that are cell-type specific in a given tumor sample. Previous studies looking at microbial reads from scRNA-seq of host cells have focused on viruses ${ }^{15,16}$. To the best of our knowledge, the only previous study to analyze bacterial reads from scRNA-seq of host cells did so in the context of known Salmonella infection using a protocol designed to capture bacterial reads ${ }^{17}$. CSI-Microbes extends upon these studies by demonstrating that viruses and intracellular bacteria that preferentially reside within one cell-type can be discovered from two commonly used scRNA-seq protocols (Smartseq2 and 10x) without knowing a priori the identity of the infecting virus or bacteria. It can do so if the input list of microbial genomes contains at least one genome with sufficient sequence similarity such that the microbe-derived reads can be aligned. In this discovery context, it is necessary to consider all microbial reads identified in the datasets, many of which are likely contaminants. Using user-specified cell-type annotations (such as those based on host transcriptomic data), CSI-Microbes identifies microbial taxa whose reads are enriched in specific cell types. Notably, this step controls for contaminating and extracellular microbes, whose 
abundances is assumed not to vary significantly between cells of different types after proper normalization.

We demonstrate that CSI-Microbes correctly identifies known intracellular microbes and their cell-type preference in datasets of immune cells exposed to the intracellular bacterium Salmonella enterica and cancer cells with known or previously reported tumor cell-specific microbes. Next, we apply it to the unexplored intracellular microbiome of non-small cell lung carcinomas where we identified tumor cell-specific intracellular bacteria in four of the thirteen tumors. By comparing infected and uninfected tumor cells, we identify a transcriptomic signature of immune down-regulation in infected tumors cells, which both supports our findings of intracellular bacteria and suggests potential mechanisms for how and why intracellular bacteria reside within tumor cells.

\section{Results}

\section{Overview of CSI-Microbes}

The inputs to CSI-Microbes are (i) a database of microbial genomes and their taxonomy tree, (ii) FASTQ files from scRNA-seq experiments, (iii) cell metadata, including cell type annotations and (iv) known covariates, such as the sequencing plate, that may be associated with differential contamination. CSI-Microbes performs two tests for the identification of cell-type specific intracellular microbes: (a) differential abundance, which compares the normalized read counts of microbial taxa between cell types, and (b) differential presence, which compares the number of cells with at least one read from microbial taxa between cell types. We use the differential presence test specifically for the analysis of sparse 10x scRNA-seq datasets, which have few microbial reads, and the differential abundance test, otherwise. The output for each patient 
sample is a list of candidate cell type-specific intracellular microbial taxa ranked by the statistical significance of their differential abundance or presence.

The algorithm proceeds in the following steps (Fig. 1, Methods): (1) Identification: scRNA-seq reads are mapped non-uniquely to microbial genomes using GATK PathSeq ${ }^{18}$ after filtering reads aligned to the host genome and spike-in transcripts (differential abundance test only). (2) Analysis: For the differential abundance test, microbial reads are normalized across cells using spike-in sequences, log-transformed and compared across specified cell types using a two-sided Wilcoxon rank-sum test with minimum average $\log _{2}$ fold-change $=.5$. The statistical significance and the area under the receiver operating curve (AUC), which is equivalent to the $U$ statistic of the Wilcoxon rank-sum test ${ }^{19}$, are used to quantify the ability of the microbial taxa to discriminate between cell types, for each microbial taxon. For the differential presence test, the number of cells with at least one UMI from the microbial taxa are compared across specific cell types using a two-sided Fisher's exact test and the statistical significance and effect size (odds ratio) are reported ${ }^{20}$. Both tests can be applied to one or more taxa at the same level or along an ancestor-descendant path in the input taxonomy tree and corrected for multiple hypotheses. Both tests are run separately for cells given their covariate annotations (plate for Smart-seq2 and sample for 10x) and combined using Stouffer's Z-score method ${ }^{21}$. (3) Validation: Post-hoc validation tests of contamination inspired by decontam ${ }^{22}$ are performed using spike-in reads and empty wells if available (Methods). These include two tests, the spike-in test and the empty wells test. The spike-in test, which is based on the observation that the number of reads from contaminating microbes are likely to correlate inversely with the sample DNA concentration, calculates the correlation between the spike-in reads and the reads of the taxon of interest. The empty wells test, which is based on the observation that contaminating sequences are more likely 
to show up in negative controls, compares the presence of microbial taxa between empty and non-empty wells.

\section{CSI-Microbes

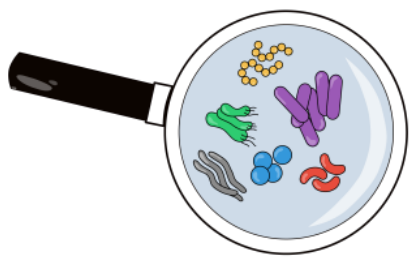

\section{Input}

Sequencing read files

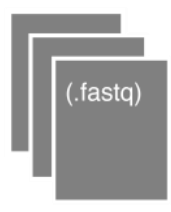

Metadata

Celltype Plate Cell

\begin{tabular}{ll|l|}
\hline Tumor & P1 & C1 \\
\hline
\end{tabular}

\begin{tabular}{ll|l|} 
Tumor & $\mathrm{P} 1$ & $\mathrm{C} 2$ \\
\hline Inmun & $\mathrm{P} 1$ & $\mathrm{C} 3$
\end{tabular}

\begin{tabular}{l|l|l|}
\hline Immune & $\mathrm{P} 1$ & $\mathrm{C} 3$ \\
\hline Immune & P1 & C4
\end{tabular}

\begin{tabular}{l|l|l|} 
Immune & P1 & C4 \\
\hline
\end{tabular}

\section{Identification}

Map reads against
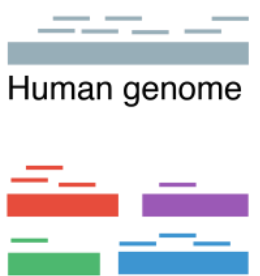

Microbe genomes

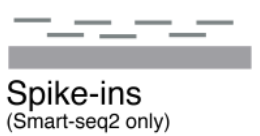

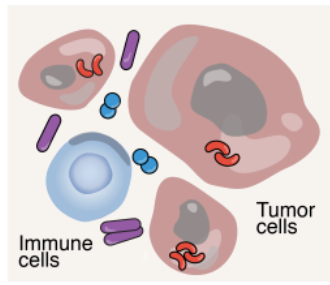

Validation

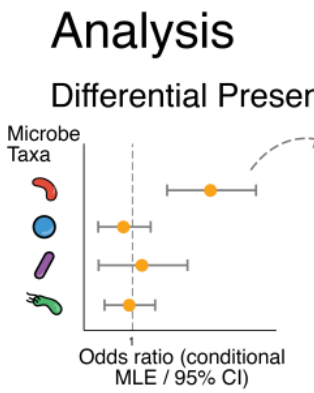

$(10 x)$

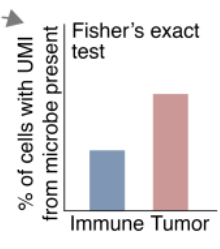

Differential Abundance (Smart-seq2)

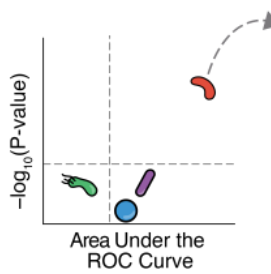

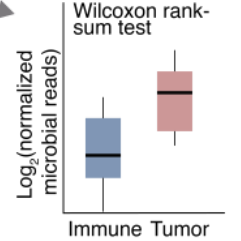

Spike-in Test

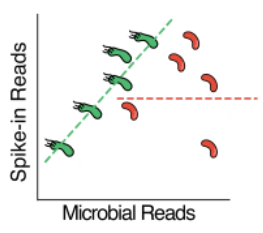

Empty Wells Test

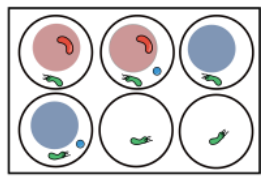

Figure 1: Overview of the CSI-Microbes approach, which takes sequencing read files (fastq format) from scRNA-seq experiments (10x or Smart-seq2) and cell metadata such as cell-type and plate (Smart-seq2) or sample (10x) as input. The steps are (1) Identification: reads are mapped to the human genome and optionally spike-in sequences (Smart-seq2 only) and unmapped reads are mapped against a large set of microbial genomes; (2) Analysis: for microbial taxa with reads identified in step 1, the $\log _{2}$ spike-in normalized reads (Differential Abundance) or the number of cells with at least one UMI (Differential Presence) are compared between user-specified cell-types (provided as input) while controlling for covariates associated with contamination (also provided as input); (3) Validation: for microbial taxa, the correlation between the number of reads from this taxa and the reads from spike-in sequences is reported (Spike-in Test) and the frequency of reads from this taxa is compared between empty wells and wells with cells (Empty Wells Test).

\section{Applying CSI-Microbes to the Salmonella gold-standard validation scRNA-seq datasets}


We validated the differential abundance test of CSI-Microbes on a "gold-standard" Smart-seq2 dataset that sequenced 262 human monocyte-derived dendritic cells (moDCs) that were exposed to either the D23580 strain or the LT2 strain of Salmonella enterica as well as 80 control cells ${ }^{23}$. The 262 Salmonella exposed cells were further labeled as 135 "infected" and 127 "bystander" cells depending on whether the presence of live, intracellular Salmonella could be detected in them using FACS. The identification step (step 1) found a median of 8,030 bacterial reads per cell that mapped to 859 bacterial genera including Salmonella. We applied the spike-in test (step 3) to the 19 most abundant genera and found that, indeed, the number of reads from the 18 genera other than the expected Salmonella is positively correlated with the number of spike-in reads, suggesting that these 18 other genera are contaminants. CSI-Microbes identified only the different microbial taxa along the path from the class Gammaproteobacteria (p-value=9e-6, $\mathrm{AUC}=.66)$ to the species Salmonella enterica $(\mathrm{p}$-value $=1 \mathrm{e}-8, \mathrm{AUC}=.70)$ as differentially abundant between the infected and bystander cells (Fig. 2A). We observed false positives when comparing cells across plates, illustrating the importance of controlling for the sequencing plate (step 2 in CSI-Microbes, Supplementary Information).

We next validated the differential presence test of CSI-Microbes on a 10x dataset in which the authors sequenced 3,485 human peripheral blood mononuclear cells (PBMCs) that were exposed to Salmonella enterica serovar Typhimurium strain SL134424. Using flow cytometry, the authors determined that $3 \%$ of the exposed PBMCs, including $90 \%$ of the monocytes, were infected with live red fluorescent protein (RFP)-expressing intracellular Salmonella. We applied CSI-Microbes to look for differentially present microbes between the monocytes and non-monocytes, which identified the path from the phylum Proteobacteria to the 
genus Salmonella. This was achieved even though only 29 UMIs mapped to bacterial genomes in this dataset (Fig. 2B).

We validated our findings of differential abundance and differential presence in the respective Salmonella datasets using two alternative mapping approaches (step 1). First, we reproduced our findings at the genera level in both datasets using the $k$-mer tool CAMMiQ ${ }^{25}$ instead of the alignment-based approach PathSeq. Second, we directly mapped unaligned reads to the Salmonella strain genome used in each experiment using the error-tolerant and ambiguitycharacter-tolerant aligner SRPRISM ${ }^{26}$ (Supplementary Information and Supplementary Fig. 1 and 2). The SRPRISM analysis of the Smart-seq2 dataset testifies to the importance of aligning reads to the entire microbial genome, instead of specific regions like the commonly used $16 \mathrm{~S}$ rRNA loci, which contain only $\sim 9 \%$ of the total reads mapped to the Salmonella strain genomes (Supplementary Information).

In these two gold-standard datasets, CSI-Microbes identified enrichments at different levels of the NCBI Taxonomy tree. In the Smart-seq2 dataset, it identified microbial taxa from the class Gammaproteobacteria to the species Salmonella enterica while in the 10x dataset, it found the path from the phylum Proteobacteria to the genus Salmonella (but not the species Salmonella enterica). To programmatically report the highest resolution microbial taxa, CSIMicrobes analyzes microbial reads from the class to the species level while performing hierarchical false discovery rate (hFDR) correction to limit the number of hypotheses ${ }^{27,28}$. 
A Infected versus Bystander cells (Smart-seq2) Taxonomy

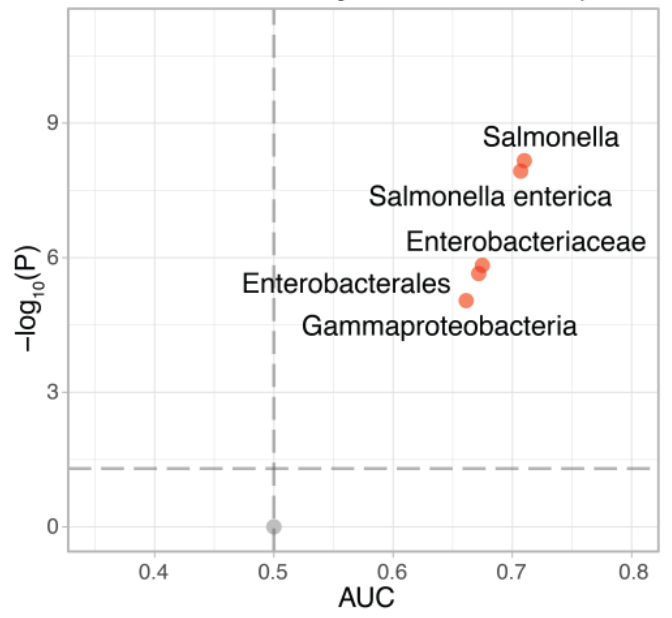

B Monocytes versus non-monocytes (10x)

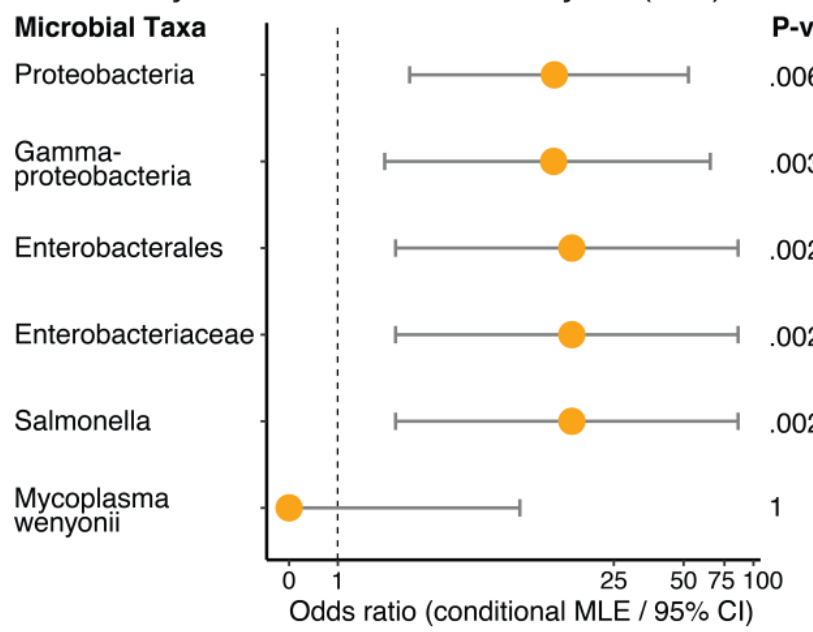

Salmonella enterica
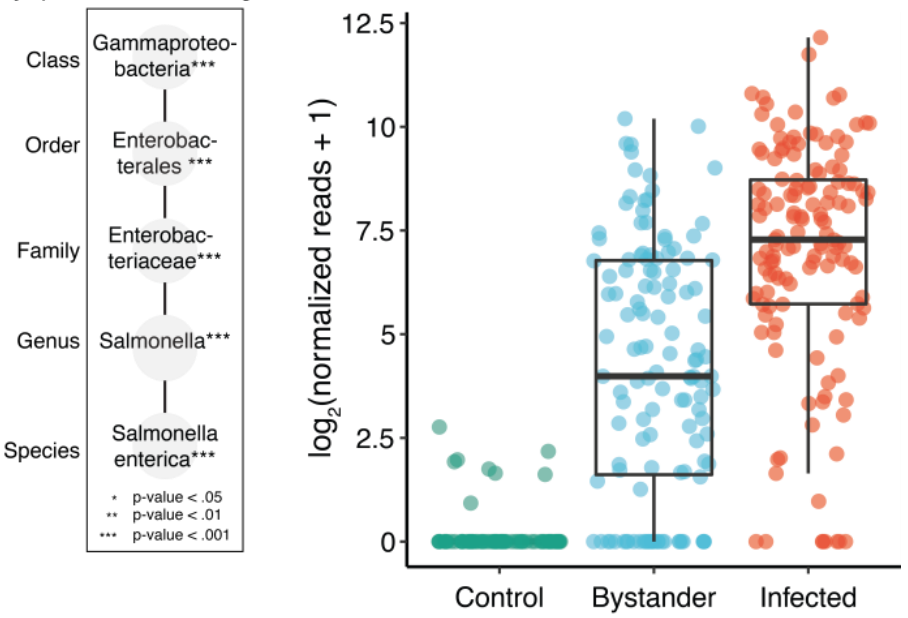

Taxonomy
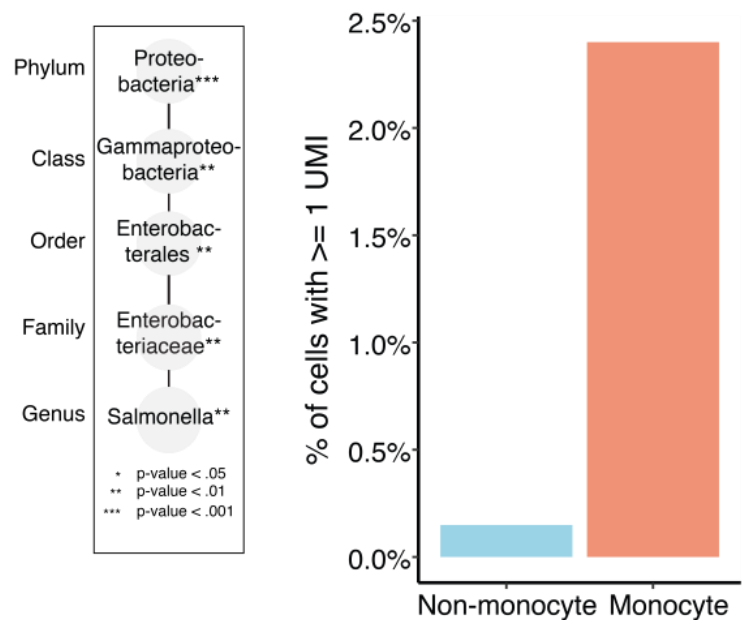

Figure 2: (A) Overview of the results from CSI-Microbes on the Smart-seq2 dataset ${ }^{23}$. The volcano plot shows the microbial taxa found to be more differentially abundant ( $p$-value $<.05$ and $A \cup C>.5$ ) in infected cells compared to bystander cells (Supplementary Table 1). The taxonomy illustration shows that all differentially abundance microbial taxa (shown on the left) are either Salmonella enterica or its taxonomic ancestors. The box plot shows that there are significantly more normalized reads mapped to Salmonella enterica in infected cells compared to bystander cells, which have significantly more normalized reads than control cells, which were not exposed to Salmonella enterica and contain almost no reads mapped to Salmonella enterica. (B) Overview of the results from CSI-Microbes on sample GSM3454529 (exposed to Salmonella enterica) from the $10 x$ dataset $^{24}$. The odds ratio plot shows that Salmonella and its taxonomic ancestors (see taxonomy illustration) but not Mycoplasma wenyonii are more differentially present in monocytes compared to non-monocytes ( $p$-value $<.05$ and odds ratio $>$ 1) (the taxonomic ancestors of Mycoplasma wenyonii, which received identical scores, were excluded for space purposes, and are listed in Supplementary Table 1). The third panel shows that UMIs from Salmonella were identified more frequently in monocytes compared to nonmonocytes. 


\section{Application of CSI-Microbes to Merkel cell and colon carcinomas}

We next validated CSI-Microbes in the context of cancer by analyzing two 10x scRNA-seq datasets from two tumor types previously reported to have tumor cell-specific intracellular microbes. Previous reports have estimated that $\sim 80 \%$ of Merkel cell carcinomas are driven by the clonal integration of the Merkel polyomavirus ${ }^{29}$. We applied CSI-Microbes to identify differentially present microbes between tumor and non-tumor cells from two Merkel cell tumors $^{30}$, which were confirmed to have integration of the Merkel polyomavirus by the original authors. In both patients, CSI-Microbes identifies the species Human polyomavirus 5, for which the only fully sequenced genome comes from the "no rank" child taxon Merkel polyomavirus, to be differentially present in tumor cells (patient 2586-4 pre-treatment: $\mathrm{p}$-value $=7 \times 10^{-9}$, odds ratio $=5.3$, post-treatment: $p$-value $=.04$, odds ratio $=6$; patient $9245-3$ : $p$-value $=10 \times 10^{-64}$, odds ratio=12.8) (Supplementary Table 2 and Supplementary Fig. 3).

The bacterial species Fusobacterium nucleatum has previously been reported to preferentially reside within colorectal carcinoma cells and to a lesser extent, stromal cells ${ }^{1}$. We applied the differential presence test of CSI-Microbes to compare tumor and non-tumor cells from a recently published 10x dataset of six colorectal carcinomas, which were not evaluated for the presence of bacteria by the authors ${ }^{31}$. In the tumor sample from patient SC028 (the only sample with UMIs aligned to Fusobacterium), CSI-Microbes identified the genus Fusobacterium ( $\mathrm{p}$-value $=.028$, odds ratio $=2.7$ ) to be more differentially present in the tumor cells compared to non-tumor cells in patient SC028 (Fig. 3A). We observed that the two cells with the most UMIs from Fusobacterium (24 and 16 UMIs respectively) were stromal cells, suggesting the presence of intracellular Fusobacterium in a small percentage of stromal cells as has been previously suggested ${ }^{1}$. CSI-Microbes also identified the bacterial species Hathewaya histolytica (p- 
value $=.01$, odds ratio=3.6) (previously called Clostridium histolyticum) to be differentially present in the tumor cells from patient SC019 (Fig. 3B and Supplementary Table 3).

Interestingly, Hathewaya histolytica has been reported to be strongly enriched in the colonic tissue of patients with ulcerative colitis compared to controls ${ }^{32}$.
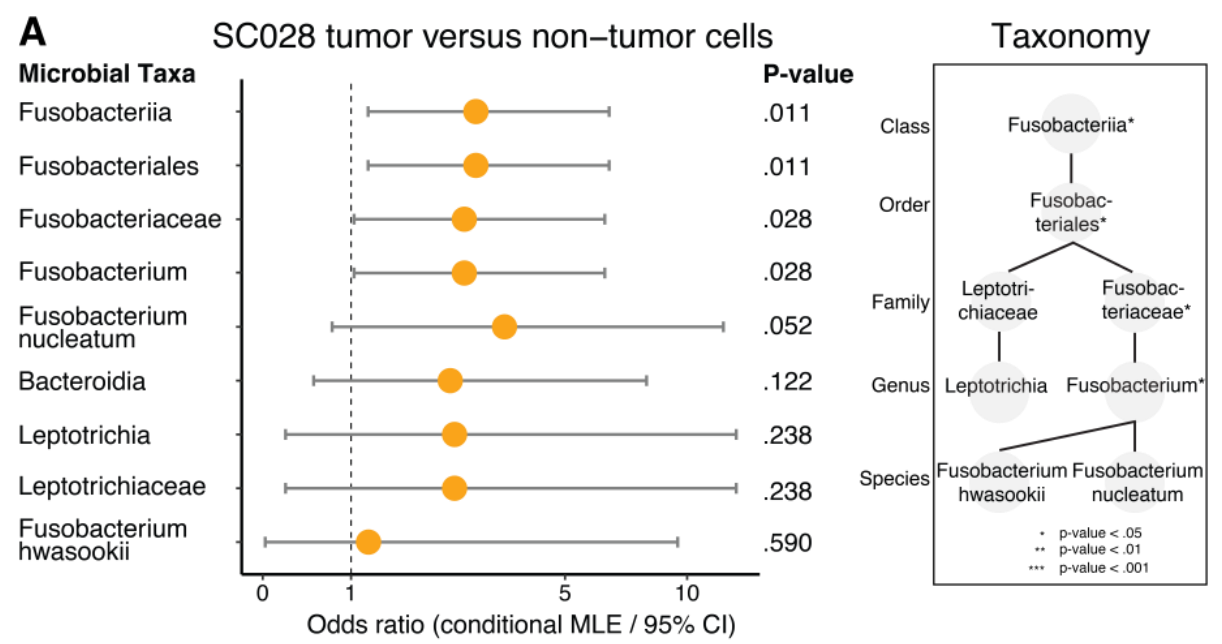

B SC019 tumor versus non-tumor cells

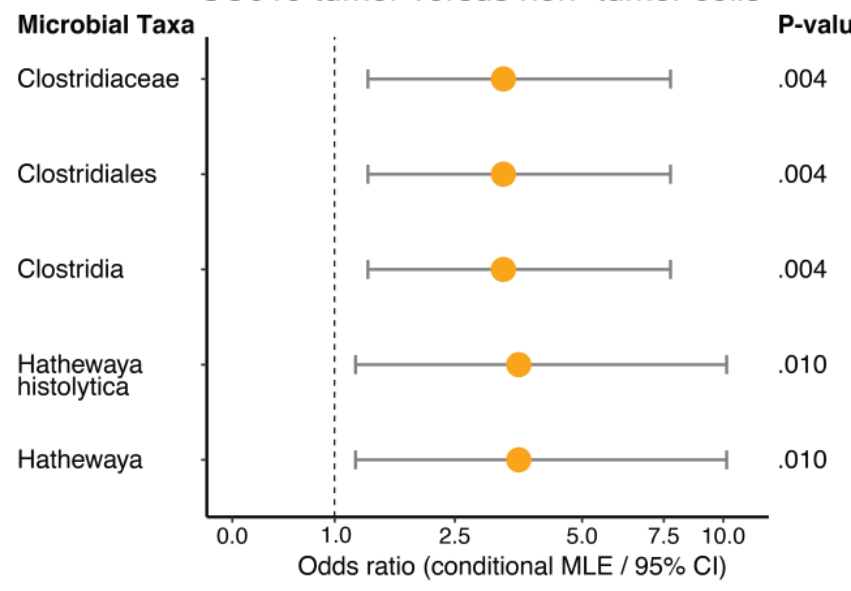

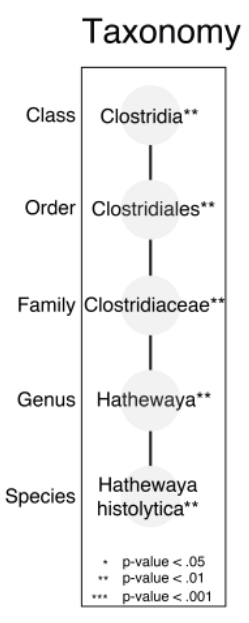

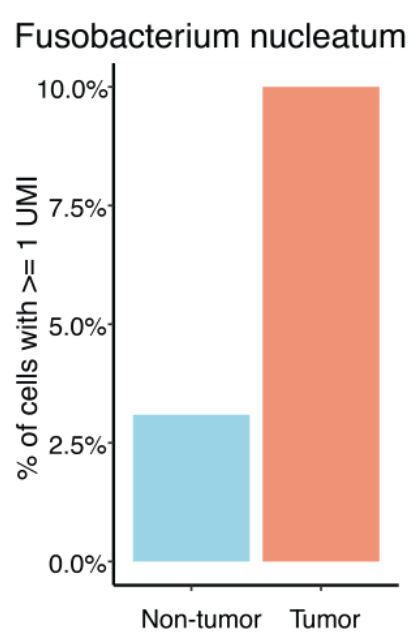

Hathewaya histolytica

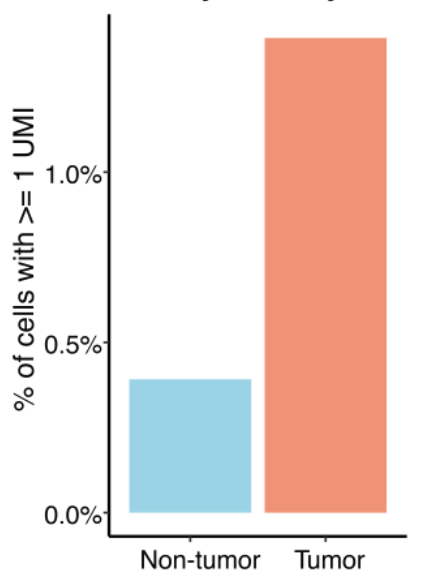

Figure 3: Overview of significant results from CSI-Microbes on colorectal carcinoma 10x dataset $^{31}$. (A) The odds ratio plot shows Fusobacterium and its taxonomic ancestors to be more differentially present in tumor cells compared to non-tumors in the tumor sample from SCO28 (see Supplementary Table 3 for more details). Although not statistically significantly, the barplot shows the difference between the percentage of tumor and non-tumor cells with at least one UMI from Fusobacterium nucleatum. (B) The odds ratio plots shows that Hathewaya histolytica and its taxonomic ancestor are more differentially present in tumor cells compared to non-tumor cells from SC019. UMIs from Hathewaya histolytica are found in a higher percentage of tumor cells compared to non-tumor cells. 


\section{Application of CSI-Microbes to lung cancer}

Next, we applied CSI-Microbes to identify differentially abundant microbes from a large, recently published lung cancer Smart-seq2 scRNA-seq dataset with spike-in sequences ${ }^{33}$. We analyzed 13 lung cancer tumors where at least 10 tumor cells and 10 non-tumor cells were sequenced in the same plate, comprising in total $\sim 11,000$ cells from 50 sequencing plates. We first compared the microbial reads (from the identification step of CSI-Microbes) to a previous analysis of the lung microbiome using $16 \mathrm{~S}_{\mathrm{rRNA}}{ }^{10}$. We identified at least one unambiguous read to 16 of the 17 species with complete genomes that were reported to be enriched in lung cancer, suggesting that Smart-seq2 data may provide sufficient coverage of the tumor microbiome (Methods). Next, using the authors' cell-type annotations (tumor, immune, stroma and epithelial), we applied CSI-Microbes to identify multiple tumors where microbial taxa are differentially abundant in tumor cells compared to immune cells (TH231, TH236, TH238, TH266; see Fig. 4A,B for examples and Supplementary Table 4 for the complete list) and stromal cells (TH236, TH266). We also detected two tumors with taxa that are differentially abundant in stromal cells (TH231; Fig. 4C) or immune cells (TH220) compared to the tumor cells. Notably, all four tumor samples containing tumor cells enriched with bacterial taxa are from tumors that had undergone at most one prior drug treatment. In contrast, the tumor sample with bacterial taxa enriched in immune cells came from a patient who had six prior lines of treatment including immunotherapy.

CSI-Microbes identified the species Cutibacterium acnes to be differentially abundant in the tumor cells compared to the immune cells in four of the thirteen tumors (TH231, TH236, TH238, TH266). Cutibacterium acnes was excluded from a previous experimental exploration of the lung tumor microbiome ${ }^{10}$ because it was identified in a large percentage of the tissue-free 
negative controls, which indicated that it is a contaminant. Consistent with this finding, we identify reads from $C$. acnes in nearly every single cell analyzed. However, $C$. acnes is significantly more abundant in tumor cells compared to immune cells in all four tumors (and is not significantly more abundant in non-tumor cells in any other tumor). Notably, C. acnes has been previously reported to exist intracellularly in epithelial cells and to be a commensal in the lung and skin as well as an opportunistic pathogen ${ }^{34-36}$. Thus, unlike bulk sequencing-based computational methods, CSI-Microbes can consider all microbes while implicitly controlling for contaminants by comparing normalized microbial reads between cells of the same patient. CSIMicrobes identifies another member of the Cutibacterium genus, Cutibacterium granulosum, which was reported to be enriched in lung cancer ${ }^{10}$, as differentially abundant in tumor cells in patient TH266 (uncorrected p-value $=.04$, Fig. 4B). Additional taxa that are differentially abundant in tumor cells include the genera Corynebacterium (TH236 and TH238) (Fig. 4A) and Staphylococcus (TH236) and the family Micrococcaceae (TH238) (Fig. 4A). In patient TH231, where CSI-Microbes found C. acnes to be enriched in tumor cells, it also identified the genus Leptotrichia to be enriched in stroma cells, which were further classified as melanocytes by the original authors, compared to non-stroma cells (Fig. 4C). In patient TH220, CSI-Microbes identified both the Micrococcus and Corynebacterium genera and the Alphaproteobacteria class to be enriched in immune cells compared to tumor cells in patient TH220. We did not find any bacterial taxa to be differentially abundant between macrophages/monocytes and any other cell type. 
A TH238 tumor versus immune cells
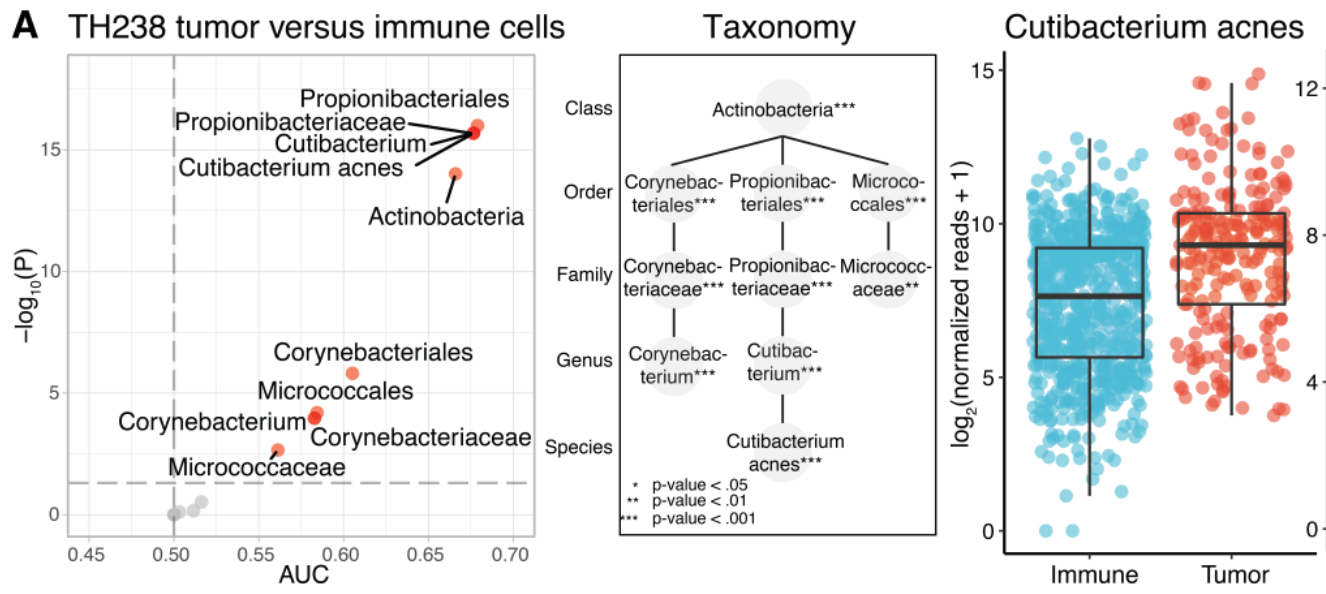

B TH266 tumor versus immune cells
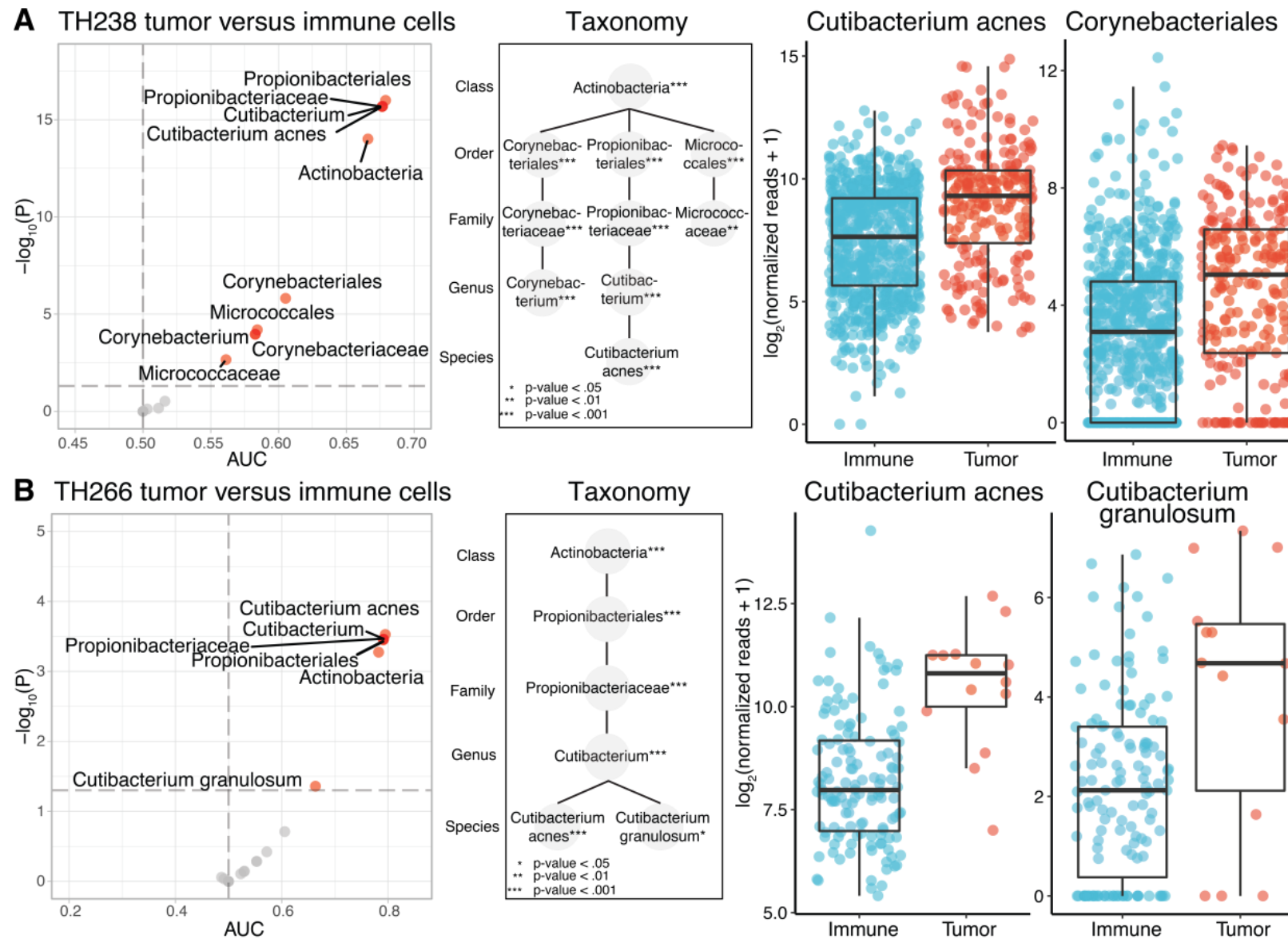

C TH231 stroma versus non-stroma cells
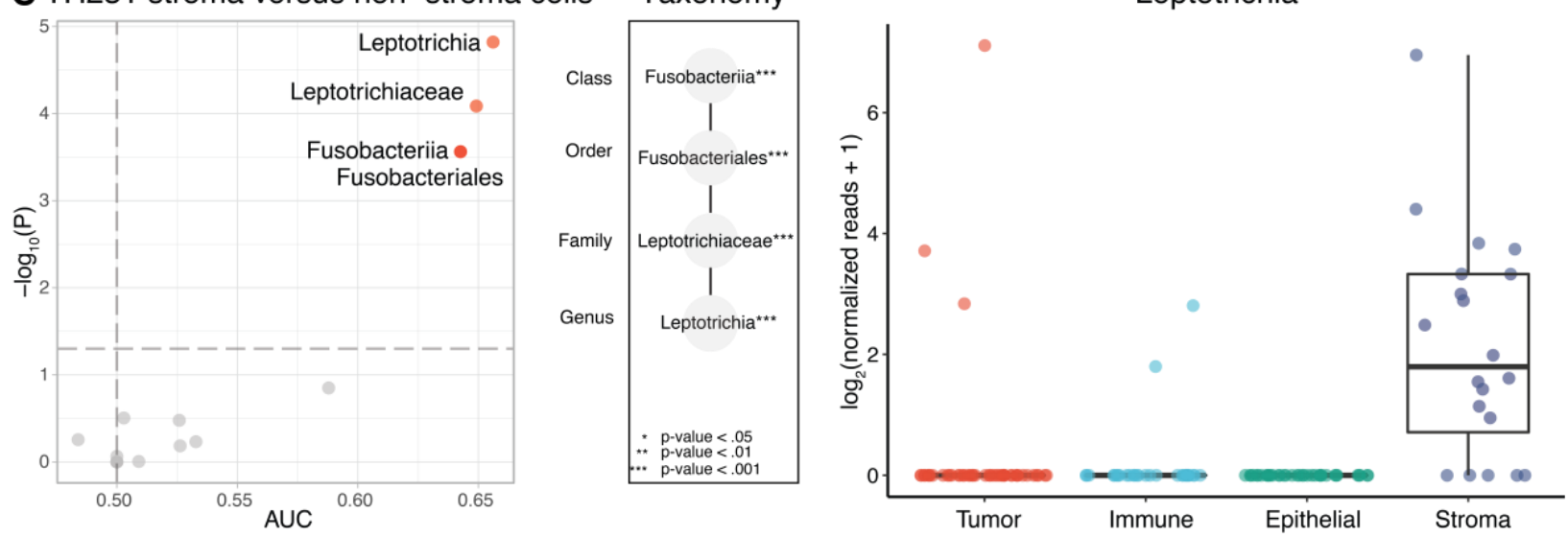

Figure 4: Key results from CSI-Microbes analysis of lung cancer (A) CSI-Microbes identified multiple bacterial taxa (all descendants of the Actinobacteria class) to be enriched in tumor cells compared to immune cells in patient TH238 (AUC> .5 and p-value <.05). (B) CSI-Microbes identified two species from the Cutibacterium genus (C. acnes and C. granulosum) to be enriched in tumor cells compared to immune cells in patient TH266. (C) CSI-Microbes identified the bacterial genus Leptotrichia to enriched in stroma cells compared to non-stroma cells in patient TH231. The boxplot shows that there are more reads to Leptotrichia in stroma cells compared to epithelial, immune and tumor cells. 
To study the transcriptomic state associated with the presence of intracellular bacteria in the lung cancer cohort that we analyzed, we performed a differential expression analysis between the tumor cells from patients TH231, TH236, TH238 and TH266 (termed "infected" because CSI-Microbes identified microbial taxa that are differentially abundant in tumor cells in each of these samples) and the tumor cells from the other patients (termed "uninfected") (Fig. 5A and Supplementary Table 5 and Methods). The most down-regulated gene in infected tumor cells compared to uninfected tumors cells is $S 100 A 9$ (FDR-corrected p-value=1e-62, AUC=.09, where for down-regulated genes 0 indicates perfect discrimination). S100A9 binds to $\$ 100 A 8$ to form a heterodimer calprotectin, which has antimicrobial properties due to its ability to sequester metal ions such as zinc, manganese and iron that are essential nutrients for microbes ${ }^{37}$. The downregulation of $5100 A 9$ thus may explain how bacteria such as $C$. acnes can survive inside these tumor cells.

Next, we performed a gene set enrichment analysis (GSEA) of the differentially expressed genes between the infected and uninfected cancer cells and clustered similar gene sets using Enrichment Map $^{38}$ (Fig. 5B and Supplementary Table 6 and Methods). The largest cluster of gene sets down-regulated in infected tumor cells contains predominately gene sets associated with processing and presentation of antigens as well as those associated with hematopoietic differentiation and response to external stimulus. This cluster is connected to the chemotaxis cluster, which includes gene sets associated with chemotaxis of leukocytes, granulocytes, and neutrophils. We observed at least three additional and unconnected downregulated gene sets that we could associate with anti-microbial response, including humoral immune response mediated by antimicrobial peptides, transition metal ion homeostasis and cell killing. Additional immune response gene sets including response to interferon gamma and 
response to interferon beta as well as interleukin-12 production are also down-regulated in the infected tumor cells. The largest cluster of up-regulated gene sets includes many gene sets associated with microtubules, which have previously been shown to be modulated by intracellular pathogens ${ }^{39}$. The association of intracellular bacteria with the down-regulation of the antigen presentation system in tumor cells, which has been previously observed in one Salmonella dataset $^{23}$ (see also Supplementary Information) is particularly relevant given the recent finding that peptides derived from bacteria can be present on the HLA class I and II molecules in melanoma ${ }^{12}$.

A Differentially expressed genes in infected tumors

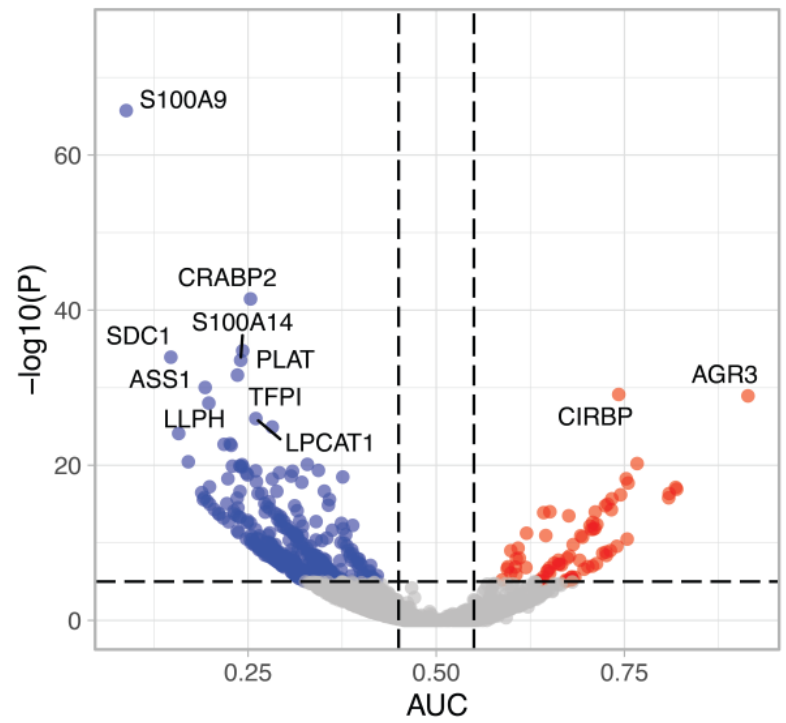

B Gene sets enriched in infected tumors

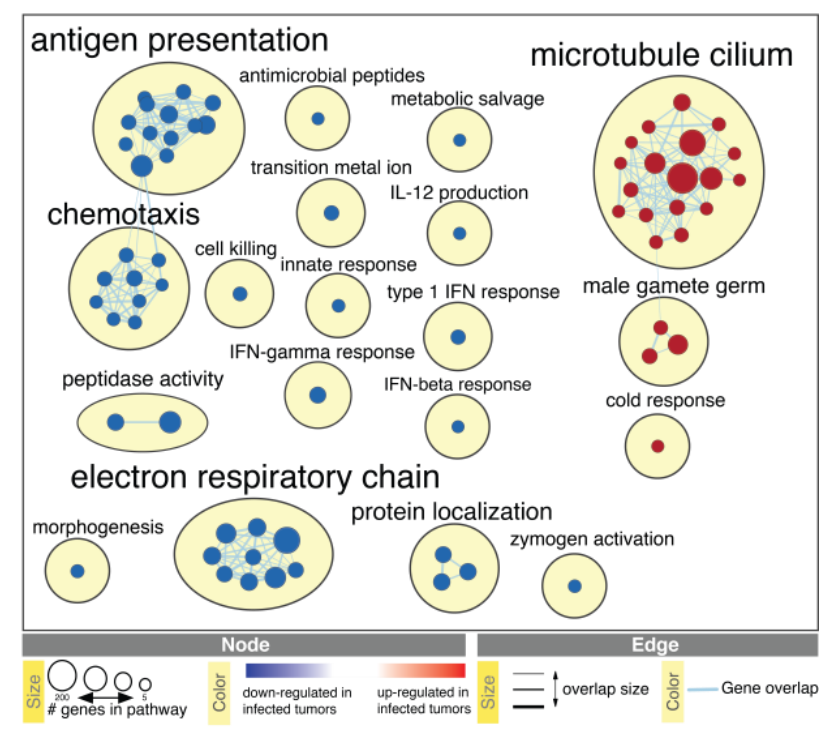

Figure 5: (A) Volcano plot of the differentially expressed genes (Supplementary Table 5) between the infected and uninfected tumors cells where blue genes (AUC $<.45$ and $p$-value < 10e-6) are significantly down-regulated in infected tumor cells and red genes (AUC > .55 and pvalue < 10e-6) are significantly up-regulated in infected tumor cells. (B) Enrichment map of the significantly enriched gene sets (FDR q-value <.02) (Supplementary Table 6) where blue nodes represent gene sets that are down-regulated in infected tumor cells and red nodes represent gene sets that are up-regulated in infected tumor cells. Gene sets are connected via edges if there are many genes in common between the two gene sets. Similar gene sets are clustered and manually named using common terms. 


\section{Discussion}

We developed and validated a first of its kind computational tool for the discovery of cell-typespecific intracellular microbes from single-cell transcriptomics data. Our approach can be applied to many existing scRNA-seq datasets without additional wet lab experiments because our framework of differential presence and abundance controls for contamination. Our bioinformatic approach is complementary to and extends existing wet-lab approaches for identifying intracellular microbes in the tumor microenvironment. Complementing HLA peptidomics, single cell transcriptomic analysis identifies not only likely intracellular bacteria but also their cell-type of residence. CSI-Microbes simultaneously searches all taxa in the input database while a staining-based approach like RNAscope ${ }^{40}$, which can identify cells containing intracellular bacteria with high confidence, is limited to using probes that are either specific to a small, predetermined set of taxa (requiring a priori knowledge) or generic to all bacteria like $16 \mathrm{~S}$ (hiding the identity of the intracellular bacteria). Intracellular microbes reported by CSI-Microbes can provide the a priori knowledge needed by approaches like RNAscope, which can and should be used to experimentally validate scRNA-seq-based findings. We also expect our approach to improve as additional tumor resident microbes are fully sequenced because many species found to be present in the tumor microbiome using $16 \mathrm{~S}$ rRNA sequencing ${ }^{10}$ lack complete reference genomes (Methods).

Beyond our specific approach, we suggest that scRNA-seq is particularly well-suited for identifying intracellular bacteria for three reasons. First, in contrast to bulk sequencing approaches, scRNA-seq protocols provide many "replicates" (in the form of single cells) from the same individual often from multiple batches, which can be used to identify and control for contaminating microbes. Second, scRNA-seq protocols are designed to sequence the RNA inside 
of single cells, which includes the RNA from any intracellular microbes (although many existing protocols, including those analyzed in this study, may under sample microbial RNA for various technical reasons). Third, scRNA-seq contains information on host transcriptomic changes associated with the presence of intracellular microbes. The findings of intracellular bacteria within tumor cells reported here and by others ${ }^{10,12}$ raise questions concerning the putative functional roles of these intracellular microbes: are they simply "innocent bystanders" and opportunistic pathogens or do they play important functional roles in tumorigenesis and response to treatment? By analyzing both human and microbial reads, we found the down-regulation of antigen processing and presentation in both moDCs infected with intracellular Salmonella (Supplementary Information) as already suggested ${ }^{23}$ and infected tumor cells in NSCLCs. These findings both provide additional support for our results and point to a potential win-win relationship between intracellular bacteria and the tumors that host them: intracellular bacteria down-regulate the antigen processing and presentation system of the host cell, which helps the tumor evade the immune system.

We focus on cancer-specific intracellular microbes, but we note that CSI-Microbes can be applied to identify intracellular microbes in other diseases. Although many intracellular bacteria preferentially invade one cell-type ${ }^{41}$, many other intracellular microbes may either not be cell-type-specific or sufficiently enriched in one cell-type to be identified by our tests. We note that the combination of our spike-in test and empty wells test could be used to identify a more general class of intracellular microbes although we did not pursue this approach because none of the datasets analyzed contained more than a small number of empty wells. In such future applications, our results underscore the importance of using spike-in sequences, empty wells, and 
multiple cell-types in the same plate to further enhance the detection accuracy of intracellular bacteria from sequencing data.

We expect that the performance of CSI-Microbes will improve as scRNA-seq technology advancements continue to increase the number of cells and sequencing depth and overcome some of the technical reasons, such as the exclusive use of polyA capture, that causes many existing protocols to under sample prokaryotic RNA molecules ${ }^{17}$. The use of polyA tail selection to enrich for polyadenylated eukaryotic mRNAs selects against prokaryotic RNA molecules, that have shorter polyA tails when polyadenylated and are less likely to be polyadenylated ${ }^{42}$. It was shown nearly thirty years ago that polyadenylation is carried out by the polymerase PAP1 in $E$. coli, which is a member of the Enterobacteriaceae family ${ }^{43}$. Although it is not known whether all bacterial taxa have polyadenylated RNAs, we hypothesize that any taxon with an orthologue of PAP1 will have some polyadenylated mRNA. It is also largely unknown which bacterial genes are polyadenylated as there are only a small number of Enterobacteriaceae genes have been shown to be polyadenylated in $E$. coli $^{44}$. Using our results from aligning the unmapped Smart-seq2 reads against the genome of Salmonella, which is also a member of the family Enterobacteriaceae, we can add to the list of likely polyadenylated genes in this family

\section{(Supplementary Table 1).}

In summary, CSI-Microbes is a powerful in silico approach for analyzing the intracellular tumor microbiome. We have shown that it identifies both known and previously reported celltype-specific intracellular bacteria and viruses as well as multiple instances of tumor-specific intracellular bacteria in NSCLCs associated with a transcriptional signature of immune and antimicrobial response down-regulation. 


\section{Methods}

\section{Data Availability}

In this study, we analyzed publicly available FASTQ files from the following datasets:

\begin{tabular}{|c|c|c|c|c|c|c|}
\hline $\begin{array}{l}\text { Sample } \\
\text { Type }\end{array}$ & $\begin{array}{l}\text { Patients } \\
\text { analyzed } \\
\text { (total) }\end{array}$ & $\begin{array}{l}\text { Cells } \\
\text { analyzed }\end{array}$ & Approach & Reference & $\begin{array}{l}\text { Spike- } \\
\text { ins }\end{array}$ & $\begin{array}{l}\text { Empty } \\
\text { Wells }\end{array}$ \\
\hline $\begin{array}{l}\text { Dendritic } \\
\text { cells } \\
\text { (exposed to } \\
\text { Salmonella) }\end{array}$ & $1(1)$ & 342 & $\begin{array}{l}\text { Smart- } \\
\text { seq2 }\end{array}$ & $\begin{array}{l}\text { NCBI BioProject } \\
\text { PRJNA437328 }\end{array}$ & $\begin{array}{l}\text { Yes } \\
(\text { ERCC) }\end{array}$ & 2 \\
\hline $\begin{array}{l}\text { PBMCs } \\
\text { (exposed to } \\
\text { Salmonella) }\end{array}$ & $1(1)$ & 3,485 & $10 x$ & $\begin{array}{l}\text { NCBI BioProject } \\
\text { PRJNA50343724 }\end{array}$ & NA & NA \\
\hline $\begin{array}{l}\text { Merkel cell } \\
\text { carcinoma }\end{array}$ & $2(2)$ & 12,754 & $10 x$ & $\begin{array}{l}\text { NCBI BioProject } \\
\text { PRJNA483959 } \\
\text { (patient 2586-4), } \\
\text { PRJNA484204 } \\
\text { (patient 9245-3) }\end{array}$ & NA & NA \\
\hline $\begin{array}{l}\text { Colorectal } \\
\text { carcinoma }\end{array}$ & $6(29)$ & 27,414 & $10 x$ & $\begin{array}{l}\text { ArrayExpress E- } \\
\text { MTAB- } 8410^{31}\end{array}$ & NA & NA \\
\hline $\begin{array}{l}\text { Non-small } \\
\text { cell lung } \\
\text { carcinoma }\end{array}$ & $13(30)$ & 10,562 & $\begin{array}{l}\text { Smart- } \\
\text { seq2 }\end{array}$ & $\begin{array}{l}\text { NCBI BioProject } \\
\text { PRJNA591860 }\end{array}$ & $\begin{array}{l}\text { Yes } \\
(\mathrm{ERCC})\end{array}$ & 0 \\
\hline
\end{tabular}

For technical reasons, 10x scRNA-seq experiments do not use spike-in sequences or empty wells. We excluded 23 of the 29 colorectal carcinomas $^{31}$ (the Korean cohort) from our analysis because the raw reads were not publicly available. We excluded 17 of the 30 non-small cell lung carcinomas (NSCLCs) ${ }^{33}$ from our analysis because either these patients did not have cells sequenced using spike-in sequences ( 2 patients) or these patients did not have at least one sequencing plate with at least ten tumor cells and ten non-tumor cells (15 patients). 


\section{Code Availability}

CSI-Microbes is logically partitioned into two modules, one module for the "identification" step and one module for the "analysis" and "validation" steps. A reproducible Snakemake 45 (https://snakemake.readthedocs.io/) workflow for identifying microbial reads from scRNA-seq datasets, which includes code to download the data from the datasets above, is available on GitHub (https://github.com/ruppinlab/CSI-Microbes-identification) although the identification module has some dependencies to the NIH Biowulf server. A reproducible Snakemake workflow for analyzing microbial reads to identify differentially abundant or differentially present microbes is available on GitHub (https://github.com/ruppinlab/CSI-Microbes-analysis). To facilitate reproduction of our analyses, we have uploaded CSI-Microbes-analysis v0.2.0 (the version used in this study) along with the relevant input files (generated by CSI-Microbesidentification v0.2.0) to Zenodo (https://doi.org/10.5281/zenodo.4695248).

\section{Smart-seq 2 datasets preprocessing}

Raw FASTQ files were trimmed using fastp ${ }^{46}$ v0.20.1 with the arguments “-unqualified_percent_limit 40 --cut_tail --low_complexity_filter --trim_poly_x". The trimmed FASTQ files were aligned to the reference human genome (GRCh38 gencode release 34) and any applicable spike-in sequences using STAR ${ }^{47}$ 2.7.6a_patch_2020-11-16 with the arguments “-soloType SmartSeq --soloUMIdedup Exact --soloStrand Unstranded --outSAMunmapped Within".

\section{0x datasets preprocessing}


Raw FASTQ files were aligned to the reference human genome using CellRanger ${ }^{48}$ v5.0.1 (https://support.10xgenomics.com/single-cell-gene-expression/software/pipelines/latest/what-iscell-ranger). The annotated polyA and template sequence oligonucleotide (TSO) sequences were trimmed, the unmapped reads were converted to the FASTQ file format trimmed and filtered using FASTP as described above before being converted to BAM files.

\section{Alignment of unmapped reads to microbial genomes}

The unaligned reads were assigned to microbial taxa using PathSeq ${ }^{18}$ v4.1.8.1

(http://software.broadinstitute.org/pathseq/) with the arguments "--filter-duplicates false--minscore-identity.7'. We constructed the reference microbial genome database by downloading the set of complete viral, bacterial and fungal genomes from RefSeq release $201^{49}$. We subsampled at least one genome from each species including any genomes annotated as either "reference genome" or "representative genome" as well as the genomes of the three Salmonella strains used in the analyzed datasets. To mitigate vector contamination, we identified regions of suspected vector contamination (including "weak" matches) in the genomes using Vecscreen_plus_taxonomy (https://github.com/aaschaffer/vecscreen plus taxonomy) with the UniVec Database (ftp://ftp.ncbi.nlm.nih.gov/pub/UniVec/.) and filtered any reads that aligned to these regions ${ }^{50}$. The alternative microbial read identification on the Salmonella datasets was performed using CAMMiQ v0.1 (https://github.com/algo-cancer/CAMMiQ) using only the above set of genomes aggregated at the genus level.

\section{Differential abundance quantification}


We define the abundance of a particular microbe in each cell to be the number of unambiguous reads assigned to the relevant genome(s) by PathSeq The abundances are normalized using the computeSpikeFactors function from scran ${ }^{51}$ v1.16.0 (https://github.com/MarioniLab/scran), which computes the library size factors using the sum of the spike-in sequences. To limit the number of hypotheses, we only test microbial taxa with counts per million microbial reads $>10$ in at least $50 \%$ of the cells from a cell-type. The logged normalized read counts are compared across cell-types using the findMarkers function from scran v1.16.0 with arguments "test = 'wilcox', lfc=0.5, block='plate". The findMarkers function from scran v1.16.0 makes inconsistent assumptions about how to distribute the values of the null distribution depending on whether the user specifies "direction $=$ "up"' or "direction $=$ 'down" ( user specifies "direction $=$ 'any", when the parameter $l f c$ is greater than zero (https://github.com/MarioniLab/scran/issues/86). The assumption for the one-sided test models our intent so we ran the comparison twice, once using with "direction='up ", and once with "direction ='down'”, selected the result with the smaller p-value for each microbial taxa and converted the one-sided $\mathrm{p}$-value to the two-sided $\mathrm{p}$-value by taking the minimum of 1 and $2 * \mathrm{p}$ value as described in a standard reference ${ }^{52}$ (page 79).

\section{Differential presence quantification}

We define the presence of a particular microbial taxon in each cell to be 1 if there are > 0 UMIs assigned unambiguously to the relevant genome(s) by PathSeq. We only analyze microbial taxa that are present in at least five cells total and at least $1 \%$ of the cells of a cell-type. We compare the presence of microbial taxa between two cell-types using the fisher.test implementation in $\mathrm{R}$ 
of Fisher's exact test with default parameters, which reports the two-sided p-value and the conditional Maximum Likelihood Estimate (MLE).

\section{False discovery rate correction}

We used two different approaches for correcting p-values for multiple hypotheses. For the CSIMicrobes results from the Salmonella dataset, we run CSI-Microbes separately for each taxonomic level and correct for the number of taxa tested at that taxonomic level using the Benjamini-Hochberg procedure ${ }^{53}$. For the CSI-Microbes results from the cancer datasets, we leveraged the finding from the Salmonella dataset that CSI-Microbes can detect differentially abundant classes. For each class, we construct the taxonomic tree using RefSeq v201 and calculate the FDR for members of that class using the hFDR.adjust function from the structSSI package $^{54}$ (https://github.com/cran/structSSI). hFDR.adjust implements the "outer-nodes" method of Yekutieli ${ }^{27}$, which is the method from that paper that is theoretically best suited for testing parent-child taxa in a taxonomic tree. To account for the multiple class hypotheses, we multiply the class-specific hFDR by the number of classes analyzed by CSI-Microbes to give the overall hierarchical FDR (hFDR). We compared the hFDR approach described above with FDR correction at the species level for the differential abundance of Salmonella enterica in the Salmonella Smart-seq2 dataset and find that the hFDR approach reports a more significant FDRcorrected p-value than the species-corrected FDR approach (1.58e-8 vs. 2.54e-8).

\section{Normalization model}

We extend the model used by decontam ${ }^{22}$ to include host and spike-in sequences such that we let the total sample RNA $(T)$ be a mixture of 3 components: human RNA $(\mathrm{H})$, spike-in RNA $(\mathrm{S})$ and 
microbial RNA (M). We can further divide the microbial RNA into contaminating microbial RNA (cM) and true microbial RNA (tM). One previously observed pattern of contaminants is the frequency of contaminating microbial RNA (cM) is likely to be inversely correlated with the human RNA concentration ${ }^{22}$. We note that the frequency of spike-in RNA is also likely to be inversely correlated with the human RNA concentration and therefore the frequency of spike-in RNA should be correlated with the frequency of contaminant RNA. Therefore, spike-in based normalization should remove any differences in the frequency of contaminating sequences between cells.

\section{Comparison to $16 \mathrm{~S}$ tumor microbiome findings}

We compared our findings of presence of bacterial taxa as numerical identifiers in NCBI's Taxonomy tree ${ }^{28}$ to previously published findings ${ }^{10}$. To do this comparison, we had to i) map the published findings to numerical taxa and to assess which of the taxa they found are in our reference database. One of the key advantages of their $16 \mathrm{~S}$ method is that it can find taxa for which there is no complete genome. In principle, CSI-Microbes can also use sub-genomic sequences in the reference database, but we chose not to use partial genomes.

In the previous study ${ }^{10}$, microbial species were presented by name, which can lead to ambiguities because there are many synonyms and the preferred genus-species name may change over time. We were able to identify NCBI Taxonomy IDs for 1,783 of the identified species. 739 of these 1,783 species have at least one completely sequenced genome and were included in our microbial database. These species included 17 of the species reported to be enriched in lung cancer $^{10}$. 


\section{Differential expression between infected and uninfected tumor cells}

We start with four groups of infected tumor cells (one group per patient) and seven groups of uninfected tumor cells (one group per patient except for TH225 and TH226, which we exclude because they have only ten and eleven cancer cells respectively). We first identified genes that are differentially expressed with an average minimum $\log _{2}$ fold-change $=.5$ between one group of infected tumor cells and one group of uninfected tumor cells, which provides 28 pair-wise comparisons (these comparisons are independent because we use a non-overlapping subset of uninfected tumor cells from each patient). We calculate a set of differentially expressed genes for each group of infected tumor cells by taking the median value of the Holm-corrected p-value for each gene (using the argument "pval.type="some" for the function findMarkers from scran ${ }^{51}$ ). Finally, the differentially expressed genes for tumor cells from each infected tumor are combined using Stouffer's Z score method ${ }^{21}$. We follow the procedure described earlier for computing the two-sided p-value.

\section{Gene set enrichment analysis}

We performed pathway enrichment analysis and visualization as previously suggested ${ }^{55}$. To perform GSEA between the infected and uninfected tumor cells, we first performed differential expression analysis as described above except that we used $\mathrm{LFC}=0$ to limit the number of genes with $p$-value $=1$ and thereby the number of tied genes. Next, we ranked genes by multiplying the $\log _{10}(\mathrm{p}$-value $)$ by -1 (AUC $>0.50$ for Wilcoxon rank sum test) or 1 (AUC $\left.\leq .50\right)$. Finally, we performed gene set enrichment analysis using the ranked genes list and the GSEAPreranked function of the GSEA tool ${ }^{56}$ v4.1.0 (http://www.gsea-msigdb.org/gsea/index.jsp) with default settings and seed=149 with the gene ontology biological processes gene set from the molecular 
signature database (MSigDB) ${ }^{56-59}$ v7.3 (http://www.gsea-msigdb.org/gsea/msigdb/index.jsp). We visualized the enriched gene sets using Enrichment Map $^{38}$ v3.3.1

(https://enrichmentmap.readthedocs.io/) with parameters: FDR q-value $<.02$ (node cutoff) and Jacard Overlap Combined Index ( $\mathrm{k}$ constant $=0.5)>.375$ (edge cutoff). Clustering was performed on the graph using MCL Cluster from clusterMaker $2^{60}$ via AutoAnnotate ${ }^{61}$ and annotated using WordCloud: Adjacent Words from AutoAnnotate ${ }^{61}$. Annotations were manually reviewed and edited where appropriate.

\section{Acknowledgements}

This research was supported in part by the Intramural Research Program of the National Institutes of Health, National Cancer Institute. WR's contribution to this research was supported in part by NSF award DGE-1632976. This work utilized the computational resources of the NIH HPC Biowulf cluster. (http://hpc.nih.gov). The authors thank Wolfgang Resch for assistance running this analysis on the NIH HPC Biowulf cluster. The authors thank Anna Aulicino, Noa Bossel Ben-Moshe, Leigh Greathouse, Hector Corrado-Bravo, Norma Andrews, Steve Christensen, Itay Tirosh, Livnat Jerby-Arnon, Yunhua Zhu, Chen Zhao, Spyros Darmanis, Frank Lowery and Sri Krishna for useful discussions about this project.

\section{Author Contributions}

Conceptualization of the project - WR AAS ER. Data curation - WR JSL KZ AAS. Formal analysis - WR. Methods - WR FS MDML RP AAS ER. Software development - WR FS EMG KZ. Software testing and documentation - WR FS AAS. Visualization - WR FS. Writing original draft - WR ER. Writing, reviewing, and editing of later drafts - WR FS EMG MDML AAS ER. Supervision - SCS AAS ER. 


\section{Competing Interests}

$\mathrm{RP}$ is a co-founder of Ocean Genomics, Inc. The other authors declare that they have no competing interests.

\section{Supplementary Tables}

Supplementary Table 1: Results from both 10x and Smart-seq2 Salmonella datasets including read counts of protein coding genes (minimum 5 reads) from LT2 and D23580 genomes and the output of CSI-Microbes for all microbial taxa from the 10x dataset (minimum 5 cells with at least one UMI) and the Smart-seq2 dataset (minimum 10 CPM in least $50 \%$ of either infected or bystander cells).

Supplementary Table 2: Output of CSI-Microbes between tumor and non-tumor cells on patients from the Merkel cell carcinoma 10x cohort ${ }^{30}$.

Supplementary Table 3: Output of CSI-Microbes between tumor and non-tumor cells on patients from the colorectal carcinoma 10x cohort ${ }^{31}$.

Supplementary Table 4: Output of CSI-Microbes between tumor and non-tumor cells on patients from the lung cancer Smart-seq2 cohort $^{33}$.

Supplementary Table 5: Complete list of differentially expressed genes between infected and uninfected lung tumor cells.

Supplementary Table 6: Up and down-regulated gene sets in infected tumor cells compared to uninfected tumor cells using GSEA.

\section{References}

1. Bullman, S. et al. Analysis of Fusobacterium persistence and antibiotic response in colorectal cancer. Science (80-. ). 358, 1443-1448 (2017).

2. Castellarin, M. et al. Fusobacterium nucleatum infection is prevalent in human colorectal carcinoma. Genome Res. 22, 299-306 (2012).

3. Gur, C. et al. Binding of the Fap2 protein of fusobacterium nucleatum to human inhibitory receptor TIGIT protects tumors from immune cell attack. Immunity 42, 344-355 (2015). 
4. Gur, C. et al. Fusobacterium nucleatum supresses anti-tumor immunity by activating CEACAM1. Oncoimmunology 8, e1581531 (2019).

5. Kostic, A. D. et al. Genomic analysis identifies association of Fusobacterium with colorectal carcinoma. Genome Res. 22, 292-298 (2012).

6. Yu, T. C. et al. Fusobacterium nucleatum Promotes Chemoresistance to Colorectal Cancer by Modulating Autophagy. Cell 170, 548-563.e16 (2017).

7. Pleguezuelos-Manzano, C. et al. Mutational signature in colorectal cancer caused by genotoxic pks + E. coli. Nature 580, 269-273 (2020).

8. Geller, L. T. et al. Potential role of intratumor bacteria in mediating tumor resistance to the chemotherapeutic drug gemcitabine. Science (80-. ). 1160, 1156-1160 (2017).

9. Poore, G. D. et al. Microbiome analyses of blood and tissues suggest cancer diagnostic approach. Nature 579, 567-574 (2020).

10. Nejman, D. et al. The human tumor microbiome is composed of tumor type-specific intracellular bacteria. Science (80-. ). 368, 973-980 (2020).

11. Abed, J. et al. Fap2 Mediates Fusobacterium nucleatum Colorectal Adenocarcinoma Enrichment by Binding to Tumor-Expressed Gal-GalNAc. Cell Host Microbe 20, 215225 (2016).

12. Kalaora, S. et al. Identification of bacteria-derived HLA-bound peptides in melanoma. Nature (2021) doi:10.1038/s41586-021-03368-8.

13. Weller, R. \& Ward, D. M. Selective recovery of $16 \mathrm{~S}$ rRNA sequences from natural microbial communities in the form of cDNA. Appl. Environ. Microbiol. 55, 1818-1822 (1989).

14. Fox, G. E., Pechman, K. R. \& Woese, C. R. Comparative cataloging of 16S ribosomal ribonucleic acid: molecular approach to procaryotic systematics. Int. J. Syst. Bacteriol. 27, 44-57 (1977).

15. Steuerman, Y. et al. Dissection of Influenza Infection In Vivo by Single-Cell RNA Sequencing. Cell Syst. 6, 679-691.e4 (2018).

16. Bost, P. et al. Host-Viral Infection Maps Reveal Signatures of Severe COVID-19 Patients. Cell 181, 1475-1488.e12 (2020).

17. Avital, G. et al. scDual-Seq: mapping the gene regulatory program of Salmonella infection by host and pathogen single-cell RNA-sequencing. Genome Biol. 18, 200 
(2017).

18. Walker, M. A. et al. GATK PathSeq: a customizable computational tool for the discovery and identification of microbial sequences in libraries from eukaryotic hosts.

Bioinformatics 34, 4287-4289 (2018).

19. Hanley, J. A. \& McNeil, B. J. The Meaning and Use of the Area under a Receiver Operating Characteristic (ROC) Curve. Radiology 143, 29-36 (1982).

20. Fisher, R. A. Statistical methods for research workers. Statistical methods for research workers, 5th ed. (Oliver and Boyd, 1934).

21. Stouffer, S. A., Suchman, E. A., DeVinney, L. C. \& Williams, R. M. The American Soldier. Adjustment During Army LIfe. (Princeton University Press, 1949).

22. Davis, N. M., Proctor, Di. M., Holmes, S. P., Relman, D. A. \& Callahan, B. J. Simple statistical identification and removal of contaminant sequences in marker-gene and metagenomics data. Microbiome 6, 226 (2018).

23. Aulicino, A. et al. Invasive Salmonella exploits divergent immune evasion strategies in infected and bystander dendritic cell subsets. Nat. Commun. 9, 4883 (2018).

24. Bossel Ben-Moshe, N. et al. Predicting bacterial infection outcomes using single cell RNA-sequencing analysis of human immune cells. Nat. Commun. 10, 3266 (2019).

25. Zhu, K. et al. Strain Level Microbial Detection and Quantification with Applications to Single Cell Metagenomics. bioRxiv (2020).

26. Morgulis, A. \& Agarwala, R. SRPRISM (Single Read Paired Read Indel Substitution Minimizer): an efficient aligner for assemblies with explicit guarantees. Gigascience $\mathbf{9}$, (2020).

27. Yekutieli, D. Hierarchical false discovery rate-controlling methodology. J. Am. Stat. Assoc. 103, 309-316 (2008).

28. Federhen, S. The NCBI Taxonomy database. Nucleic Acids Res. 40, D136-143 (2012).

29. Feng, H., Shuda, M., Chang, Y. \& Moore, P. S. Clonal integration of a polyomavirus in human Merkel cell carcinoma. Science (80-. ). 319, 1096-1100 (2008).

30. Paulson, K. G. et al. Acquired cancer resistance to combination immunotherapy from transcriptional loss of class I HLA. Nat. Commun. 9, (2018).

31. Lee, H. O. et al. Lineage-dependent gene expression programs influence the immune landscape of colorectal cancer. Nat. Genet. 52, 594-603 (2020). 
32. Kleessen, B., Kroesen, A. J., Buhr, H. J. \& Blaut, M. Mucosal and invading bacteria in patients with inflammatory bowel disease compared with controls. Scand. J.

Gastroenterol. 37, 1034-1041 (2009).

33. Maynard, A. et al. Therapy-Induced Evolution of Human Lung Cancer Revealed by Single-Cell RNA Sequencing. Cell 182, 1232-1251.e22 (2020).

34. Mayslich, C., Grange, P. A. \& Dupin, N. Cutibacterium acnes as an opportunistic pathogen: An update of its virulence-associated factors. Microorganisms 9, 1-21 (2021).

35. Bae, Y. et al. Intracellular propionibacterium acnes infection in glandular epithelium and stromal macrophages of the prostate with or without cancer. PLoS One 9, e90324 (2014).

36. Eishi, Y. Etiologic link between sarcoidosis and Propionibacterium acnes. Respir. Investig. 51, 56-68 (2013).

37. Brophy, M. B. \& Nolan, E. M. Manganese and microbial pathogenesis: Sequestration by the mammalian immune system and utilization by microorganisms. ACS Chem. Biol. 10, 641-651 (2015).

38. Merico, D., Isserlin, R., Stueker, O., Emili, A. \& Bader, G. D. Enrichment Map: A Network-Based Method for Gene-Set Enrichment Visualization and Interpretation. PLoS One 5, e13984 (2010).

39. Radhakrishnan, G. K. \& Splitter, G. A. Modulation of host microtubule dynamics by pathogenic bacteria. Biomol. Concepts 3, 571-580 (2012).

40. Wang, F. et al. RNAscope: A novel in situ RNA analysis platform for formalin-fixed, paraffin-embedded tissues. J. Mol. Diagnostics 14, 22-29 (2012).

41. Silva, M. T. Classical labeling of bacterial pathogens according to their lifestyle in the host: Inconsistencies and alternatives. Front. Microbiol. 3, 71 (2012).

42. Mohanty, B. K. \& Kushner, S. R. Bacterial/archaeal/organellar polyadenylation. Wiley Interdiscip Rev RNA 2, 256-276 (2010).

43. Cao, G. J. \& Sarkar, N. Identification of the gene for an Escherichia coli poly(A) polymerase. Proc. Natl. Acad. Sci. U. S. A. 89, (1992).

44. Régnier, P. \& Marujo, P. E. Polyadenylation and Degradation of RNA in Prokaryotes. in Madame Curie Bioscience Database [Internet] (Landes Bioscience, 2013).

45. Köster, J. \& Rahmann, S. Snakemake-a scalable bioinformatics workflow engine. Bioinformatics 28, 2520-2522 (2012). 
46. Chen, S., Zhou, Y., Chen, Y. \& Gu, J. Fastp: An ultra-fast all-in-one FASTQ preprocessor. Bioinformatics 34, i884-i890 (2018).

47. Dobin, A. et al. STAR: Ultrafast universal RNA-seq aligner. Bioinformatics 29, 15-21 (2013).

48. Zheng, G. X. Y. et al. Massively parallel digital transcriptional profiling of single cells. Nat. Commun. 8, 14049 (2017).

49. O'Leary, N. A. et al. Reference sequence (RefSeq) database at NCBI: Current status, taxonomic expansion, and functional annotation. Nucleic Acids Res. 44, D733-D745 (2016).

50. Schäffer, A. A. et al. VecScreen-plus-taxonomy: Imposing a tax(onomy) increase on vector contamination screening. Bioinformatics 34, 755-759 (2018).

51. Lun, A. T. L., Mccarthy, D. J. \& Marioni, J. C. A step-by-step workflow for low-level analysis of single-cell RNA-seq data with Bioconductor [ version 2 ; referees : 3 approved , 2 approved with reservations ]. F1000Research 5, (2016).

52. Sokal, R. R. \& Rohlf, F. J. Biometry. (W. H. Freeman, 1995).

53. Benjamini, Y.\& Hochberg, Y. Controlling the False Discovery Rate : A Practical and Powerful Approach to Multiple Testing. J. R. Stat. Soc. 57, 289-300 (1995).

54. Sankaran, K. \& Holmes, S. structSSI: Simultaneous and selective inference for grouped or hierarchically structured data. J. Stat. Softw. 59, 1-21 (2014).

55. Reimand, J. et al. Pathway enrichment analysis and visualization of omics data using g:Profiler, GSEA, Cytoscape and EnrichmentMap. Nat. Protoc. 22, 924-934 (2019).

56. Subramanian, A., Tamayo, P., Mootha, V. K., Mukherjee, S. \& Ebert, B. L. Gene set enrichment analysis : A knowledge-based approach for interpreting genome-wide. Proc. Natl. Acad. Sci. 102, 15545-15550 (2005).

57. Ashburner, M. et al. Gene ontology: Tool for the unification of biology. Nat. Genet. 25, 25-29 (2000).

58. Carbon, S. et al. The Gene Ontology resource: Enriching a GOld mine. Nucleic Acids Res. 49, D325-D334 (2021).

59. Liberzon, A. et al. Molecular signatures database (MSigDB) 3.0. Bioinformatics 27, 1739-1740 (2011).

60. Morris, J. H. et al. ClusterMaker: A multi-algorithm clustering plugin for Cytoscape. 
BMC Bioinformatics 12, (2011).

61. Kucera, M., Isserlin, R., Arkhangorodsky, A. \& Bader, G. D. AutoAnnotate: A Cytoscape app for summarizing networks with semantic annotations [version 1; peer review: 2 approved]. F1000Research 5, (2016). 


\section{CSI-Microbes}
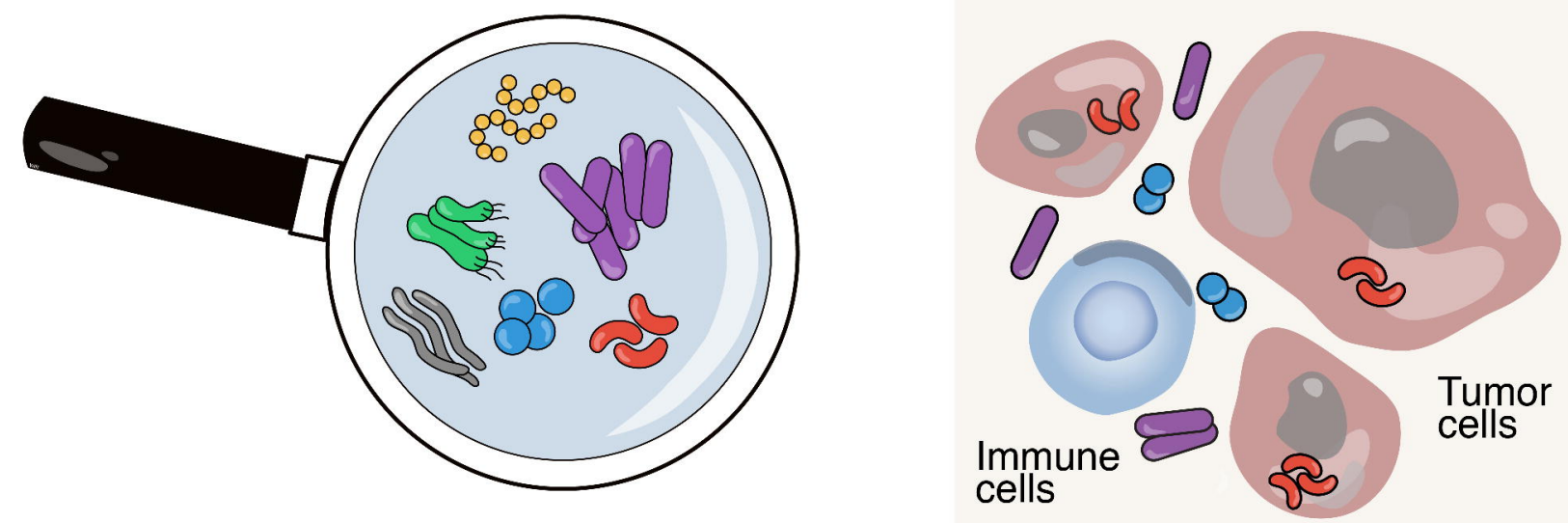

Input

Sequencing read files

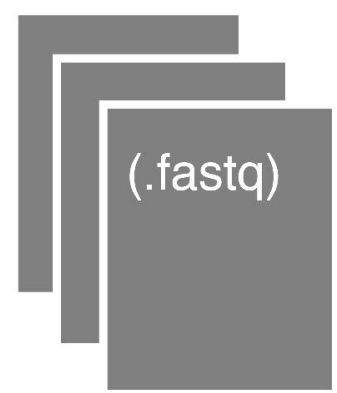

Metadata

Celltype Plate Cell Tumor $\mathrm{P1}$ C1 \begin{tabular}{l|l|l} 
Tumor & $\mathrm{P} 1$ & $\mathrm{C} 2$
\end{tabular}

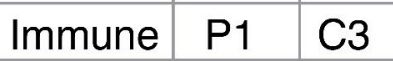
\begin{tabular}{l|l|l} 
Immune & $\mathrm{P} 1$ & $\mathrm{C} 4$
\end{tabular}

\section{Identification Analysis}

Map reads against
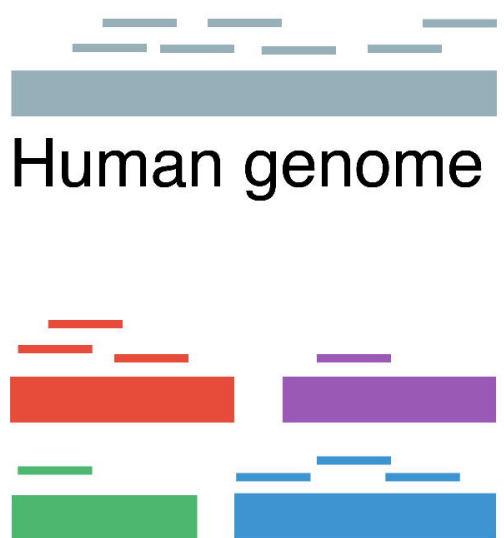

Microbe genomes

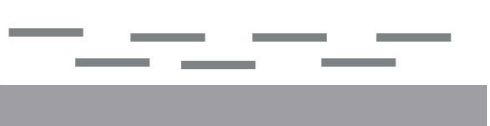

Spike-ins

(Smart-seq2 only)
Differential Presence (10x)

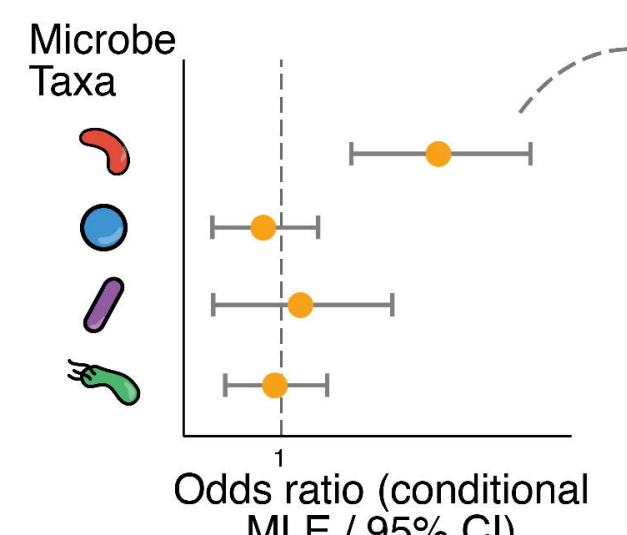

MLE / $95 \% \mathrm{Cl}$ )

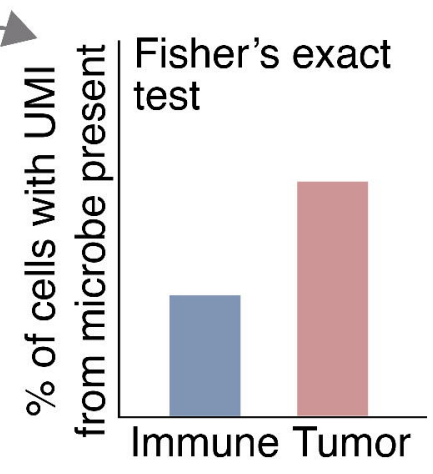

Differential Abundance (Smart-seq2)

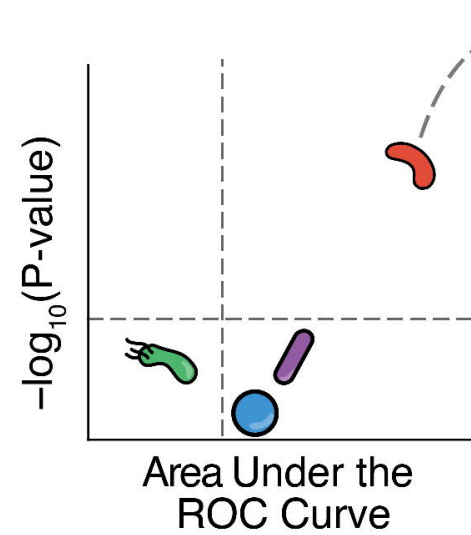

Wilcoxon rank-

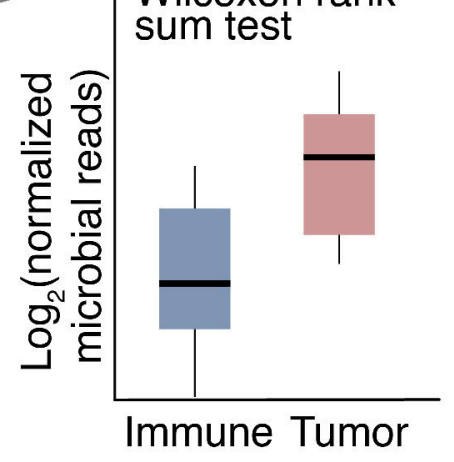

Validation

Spike-in Test

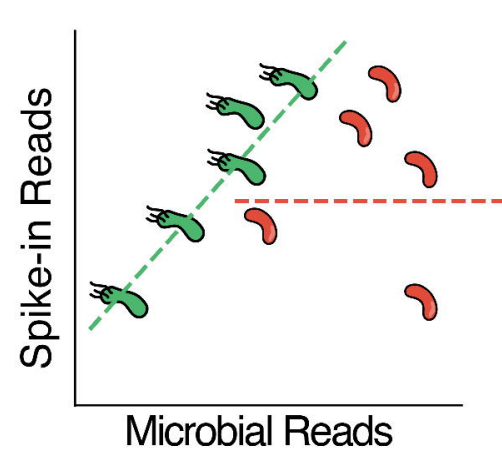

Empty Wells Test

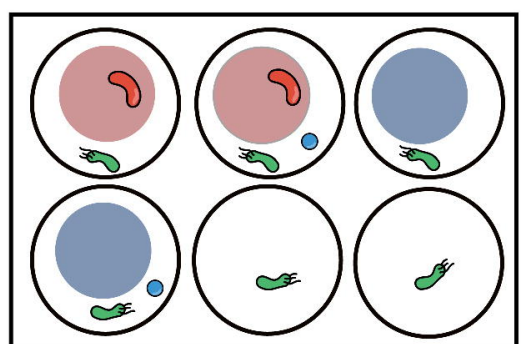


A Infected versus Bystander cells (Smart-seq2) Taxonomy

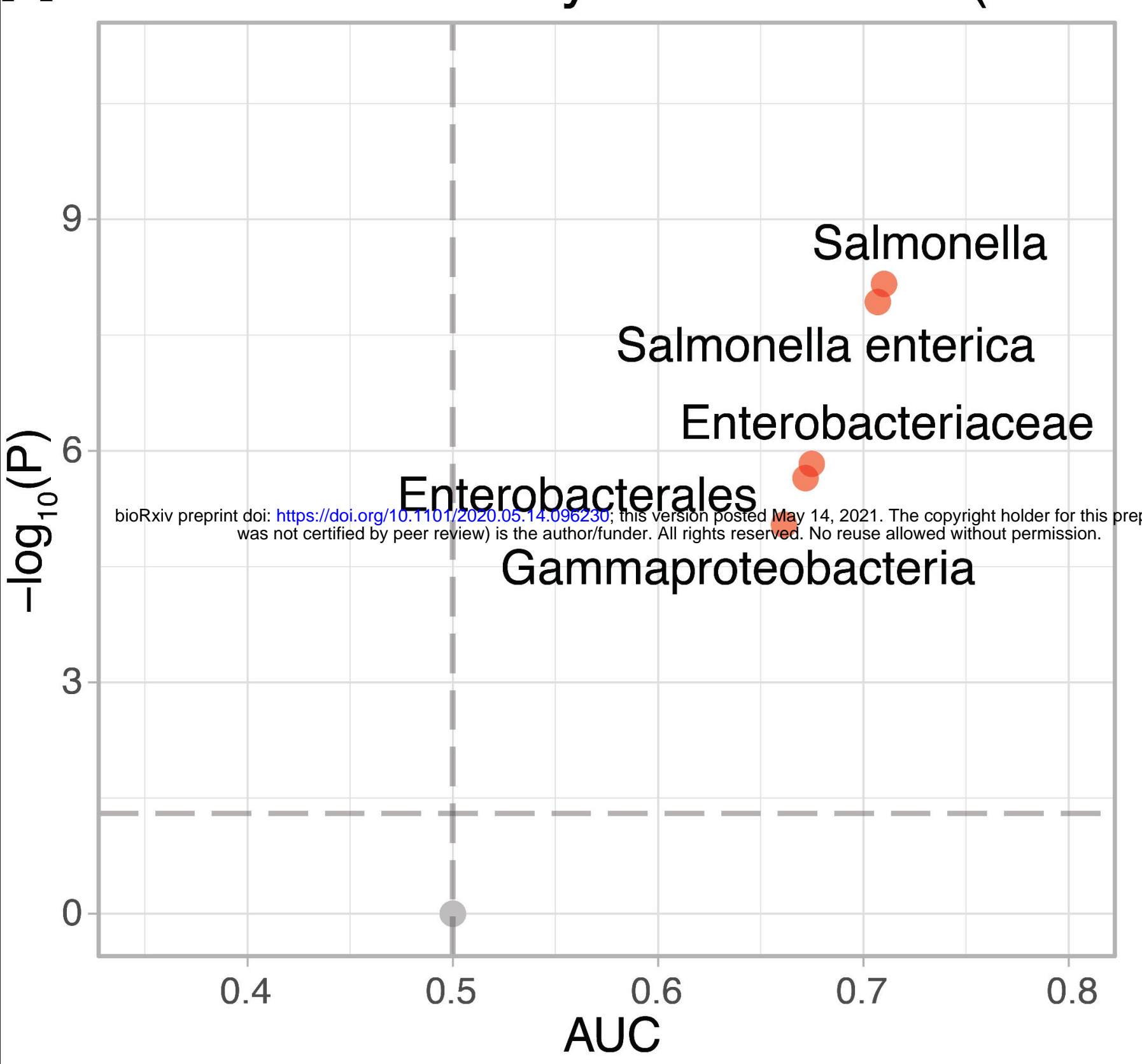

B Monocytes versus non-monocytes (10x) Microbial Taxa

Proteobacteria

Gamma-

proteobacteria

Enterobacterales

Enterobacteriaceae

Salmonella

Mycoplasma wenyonii

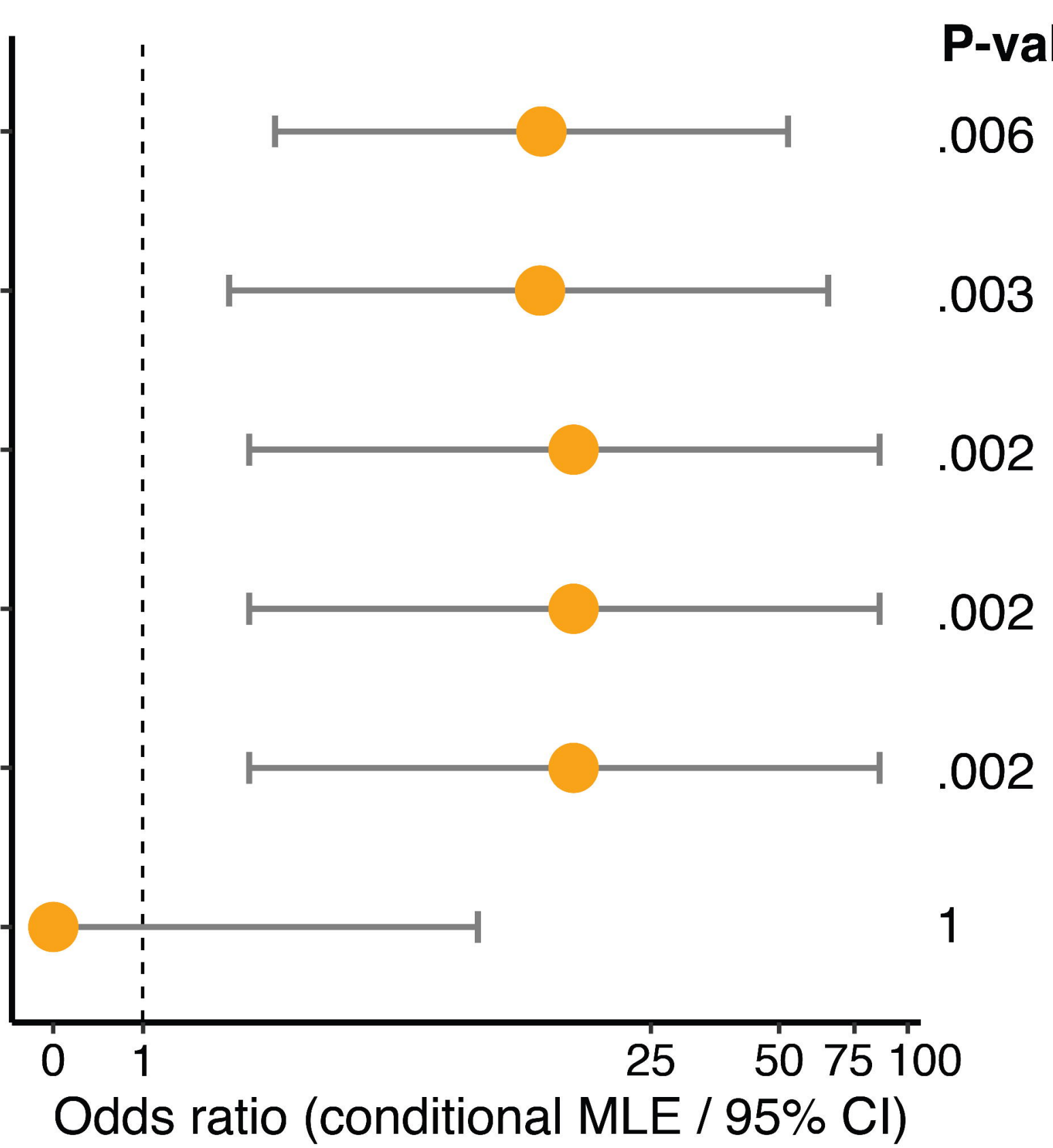

Salmonella enterica
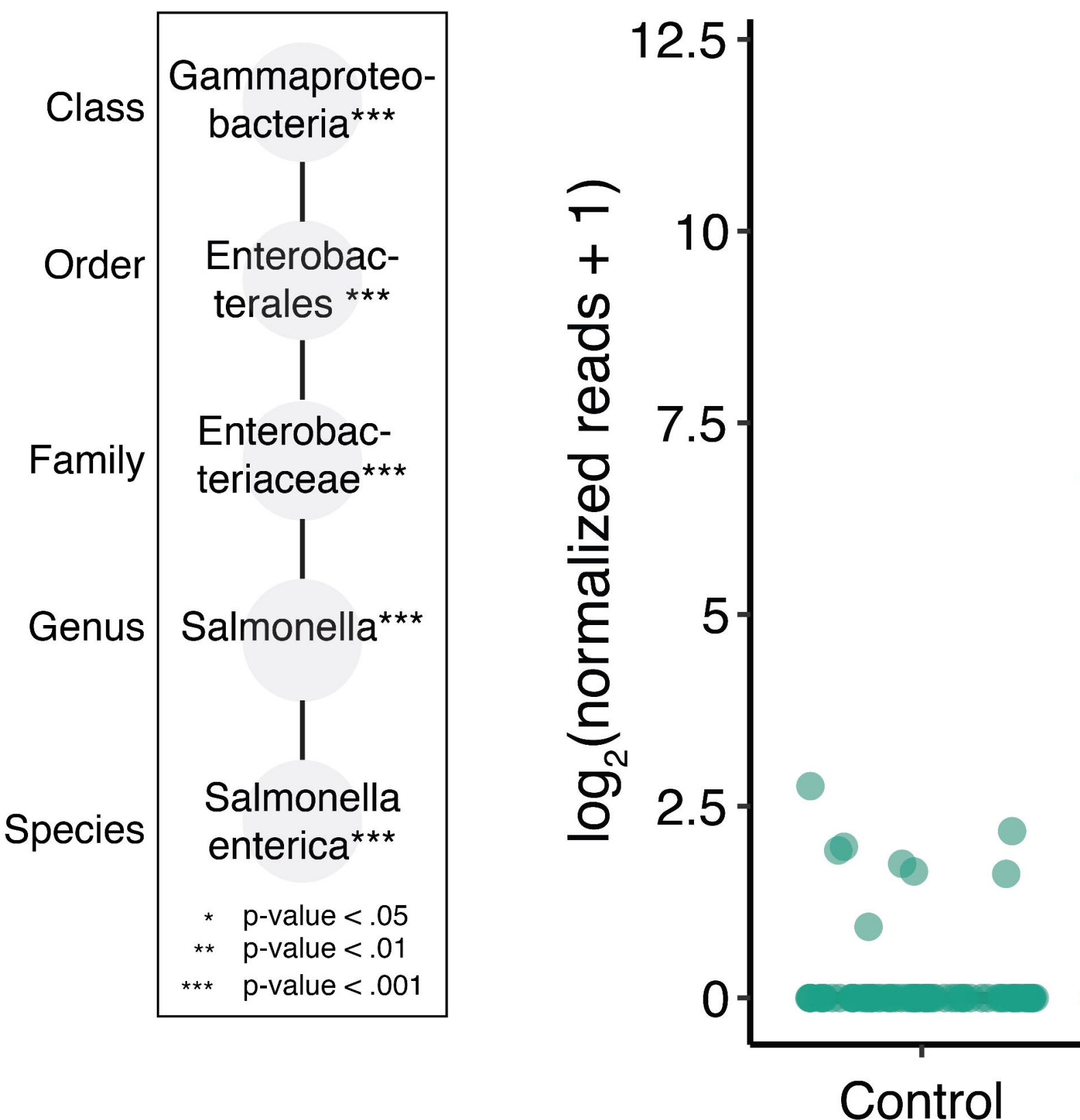

Taxonomy

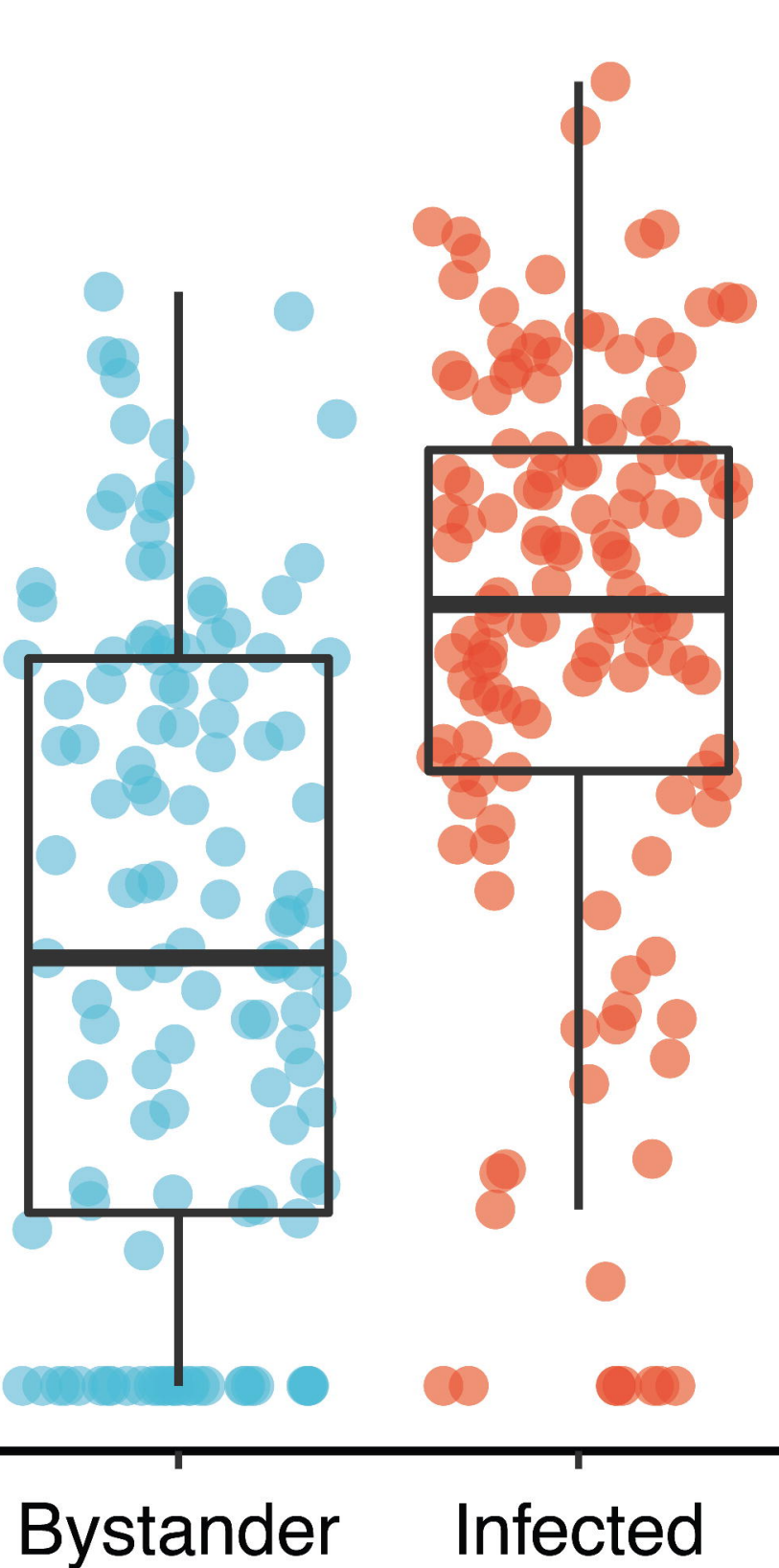

Salmonella
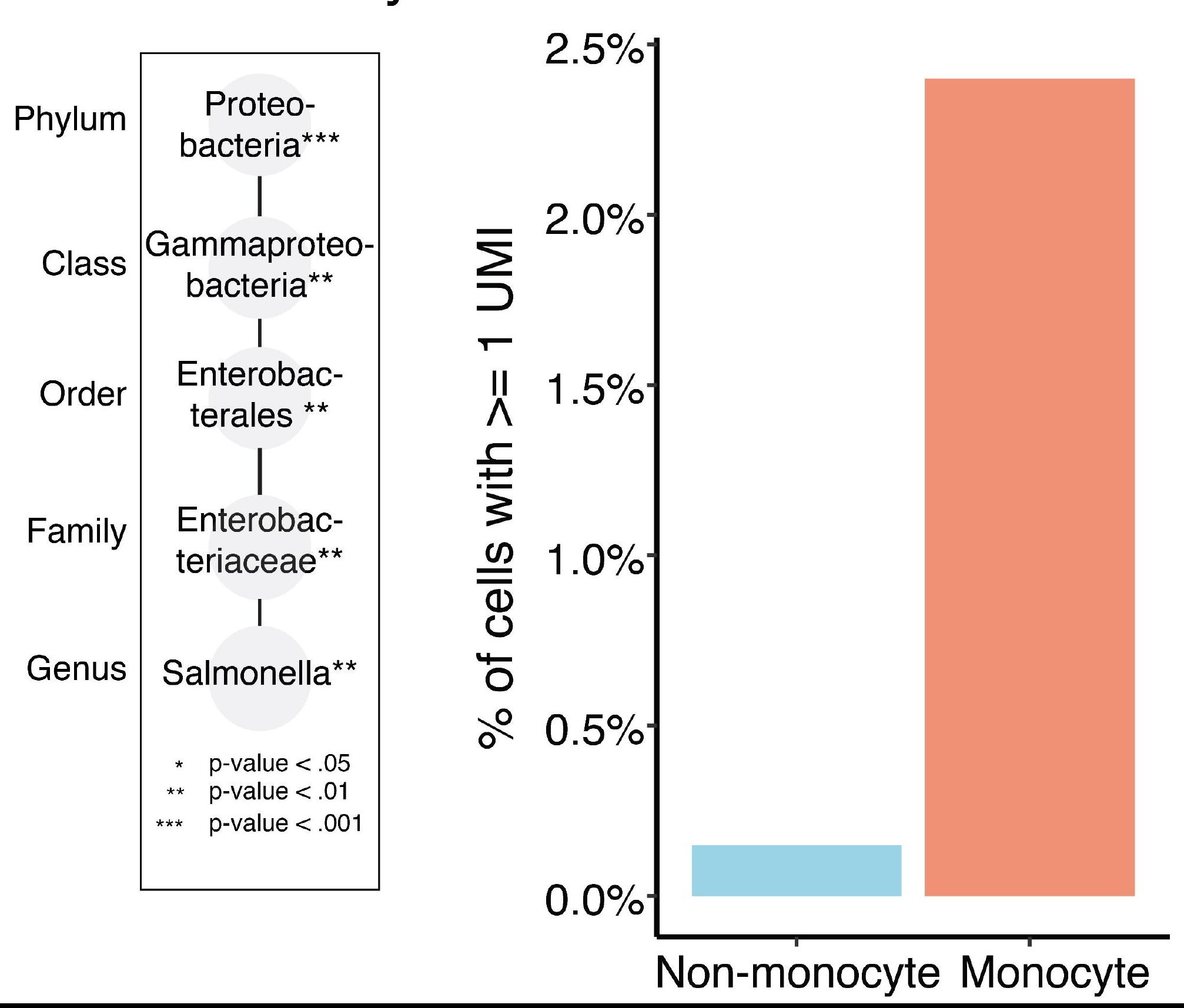


\section{A} Microbial Taxa

Fusobacteriia

Fusobacteriales

Fusobacteriaceae

Fusobacterium

Fusobacterium nucleatum

Bacteroidia

Leptotrichia

Leptotrichiaceae

Fusobacterium

hwasookii

SC028 tumor versus non-tumor cells

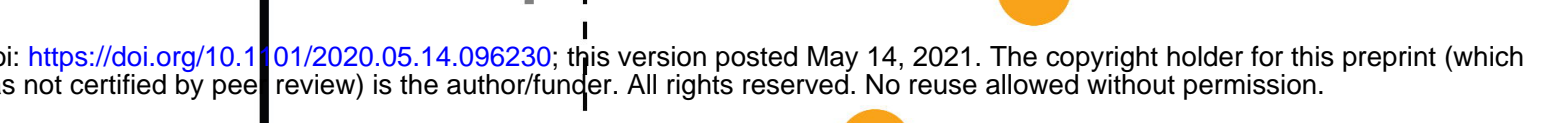

B Microbial Taxa

Clostridiaceae

Clostridiales

Clostridia

Hathewaya histolytica

Hathewaya

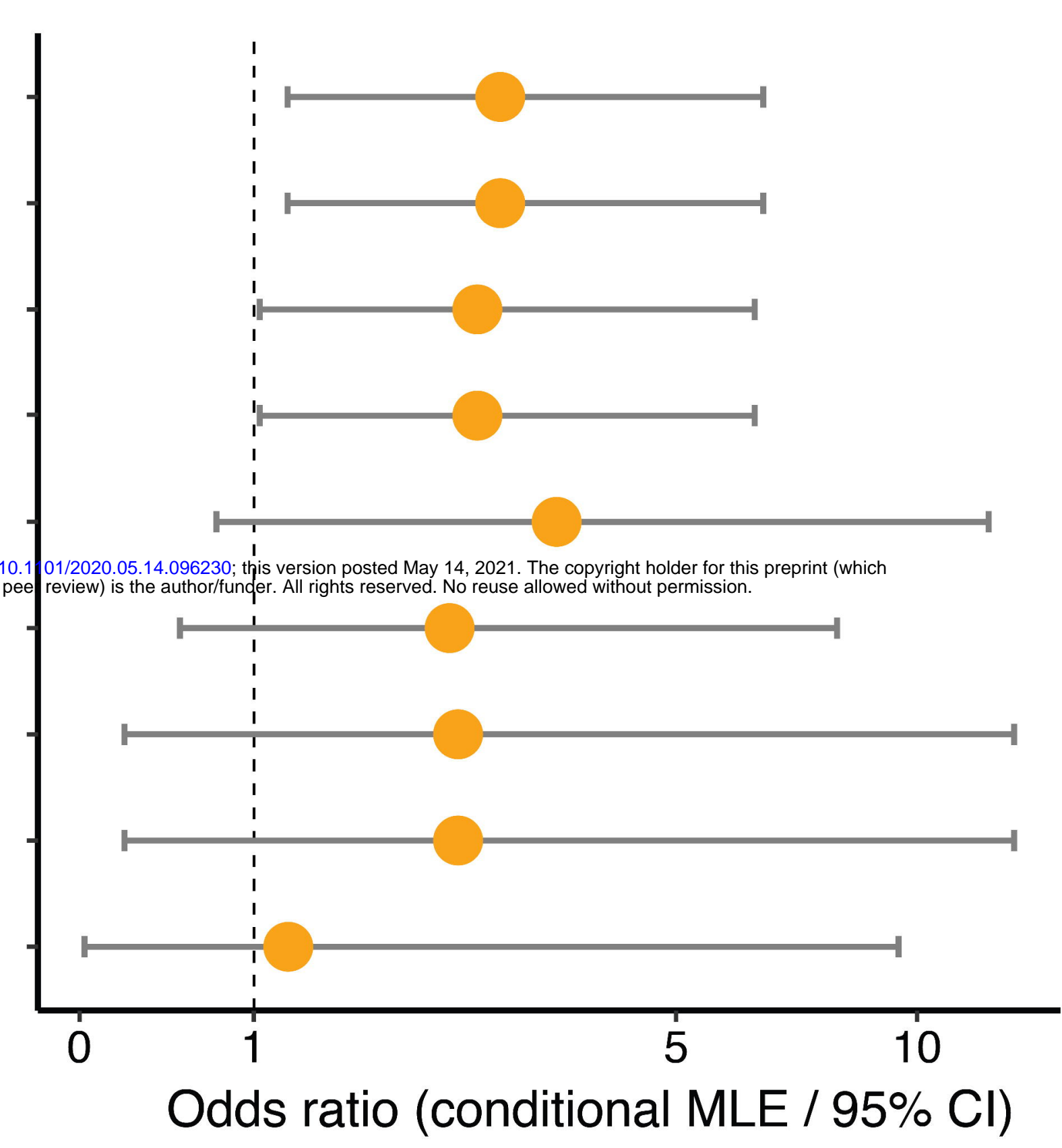

SC019 tumor versus non-tumor cells

P-value

.011

.011

.028

.028

.052

.122

.238

.238

.590
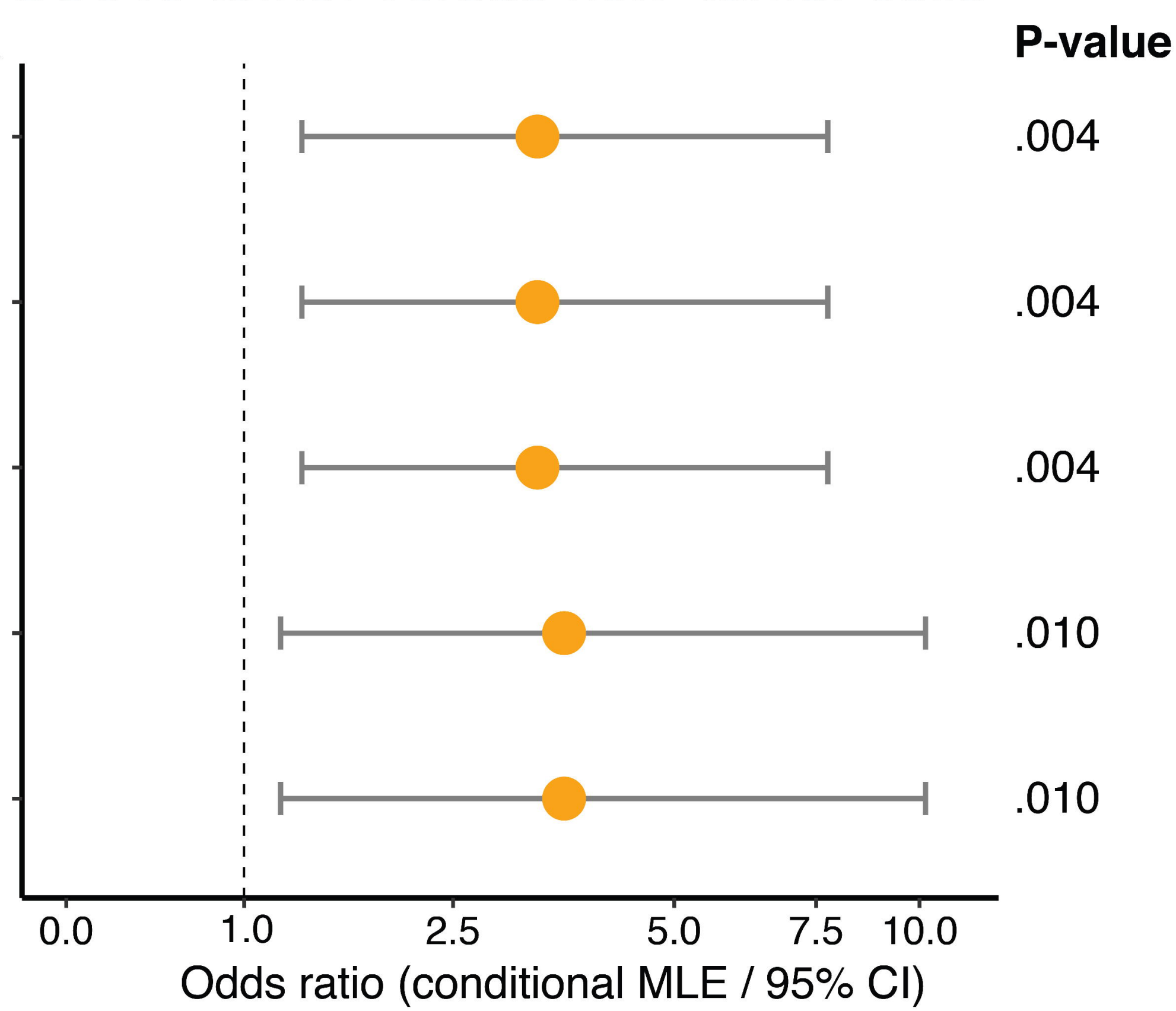

Taxonomy

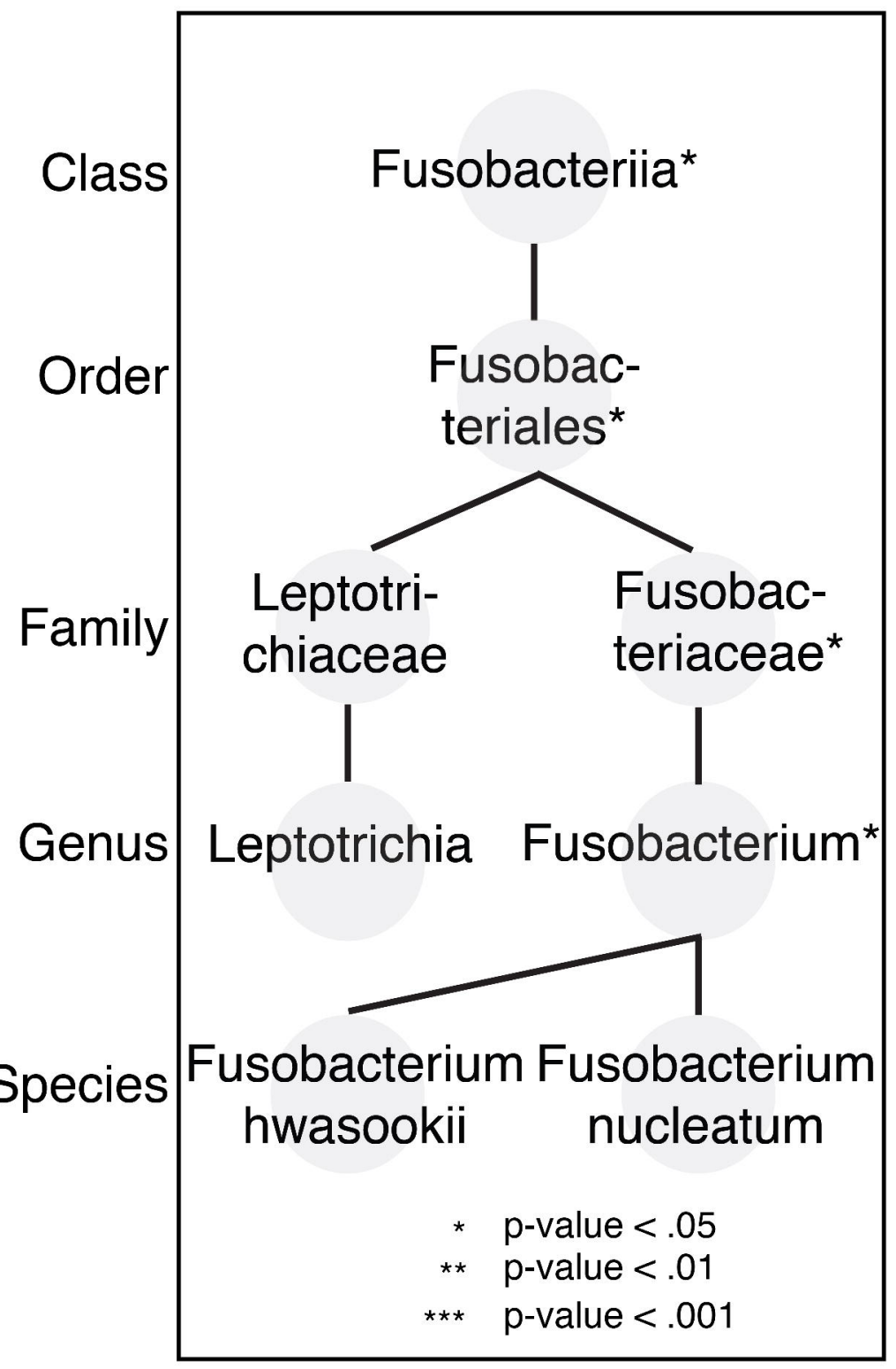

Taxonomy

.004

.004

.004

.010

.010

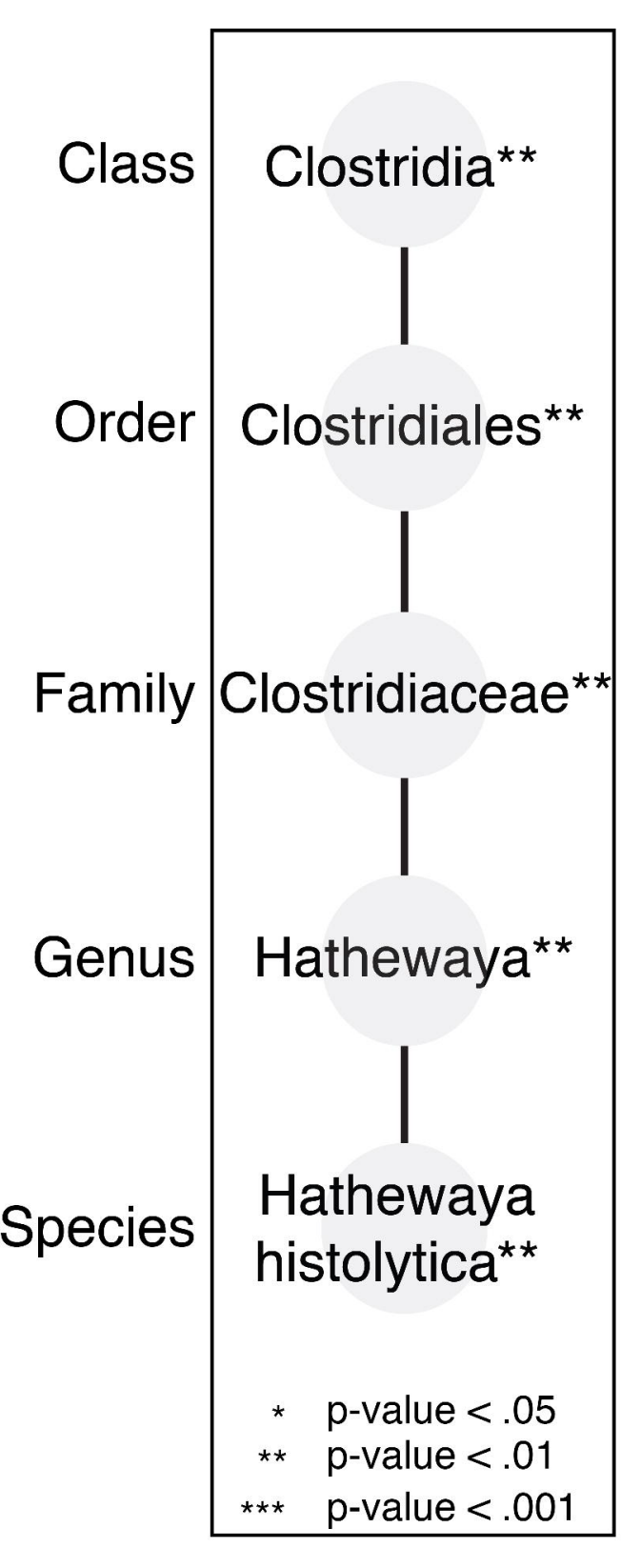

Fusobacterium nucleatum

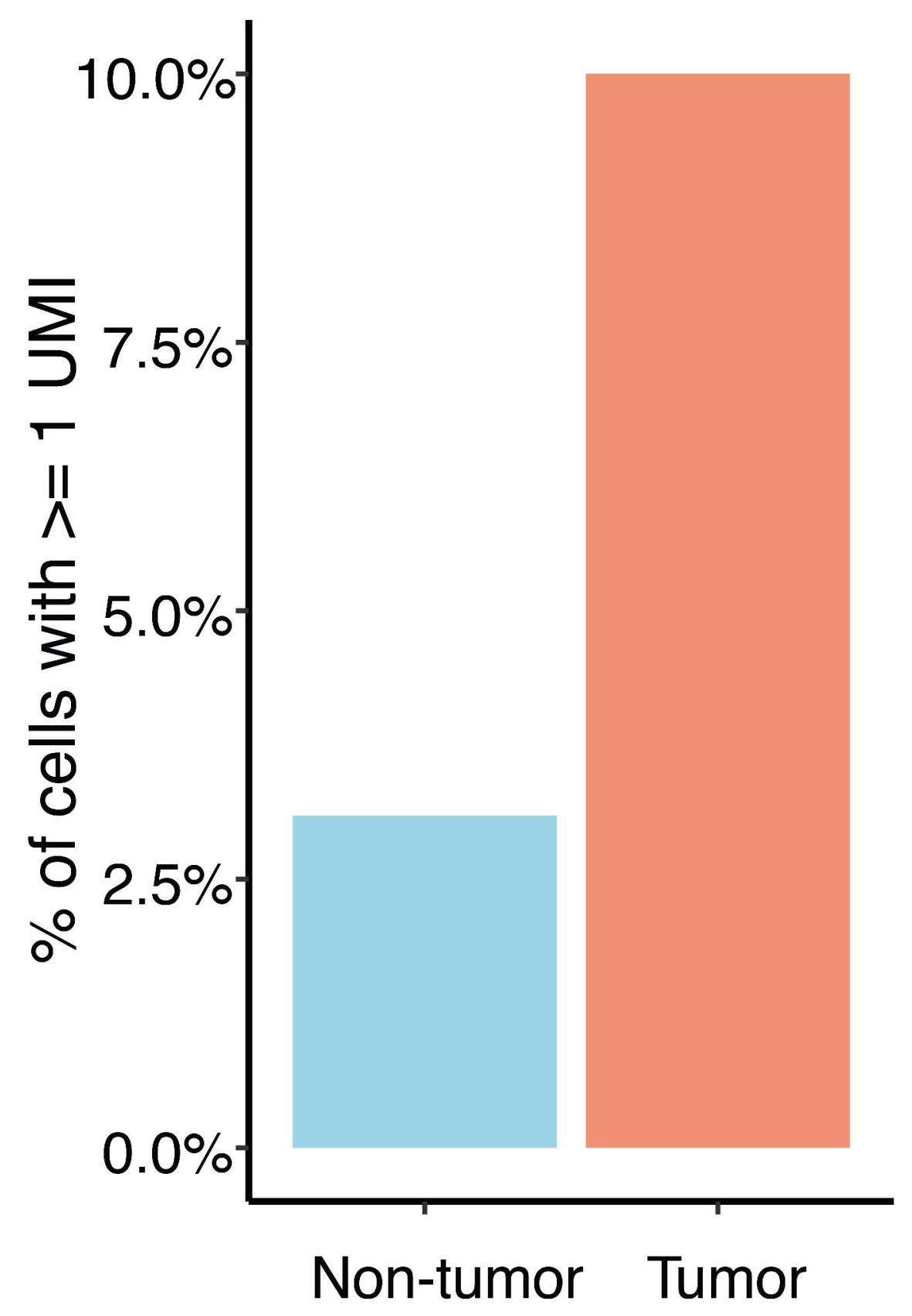

Hathewaya histolytica

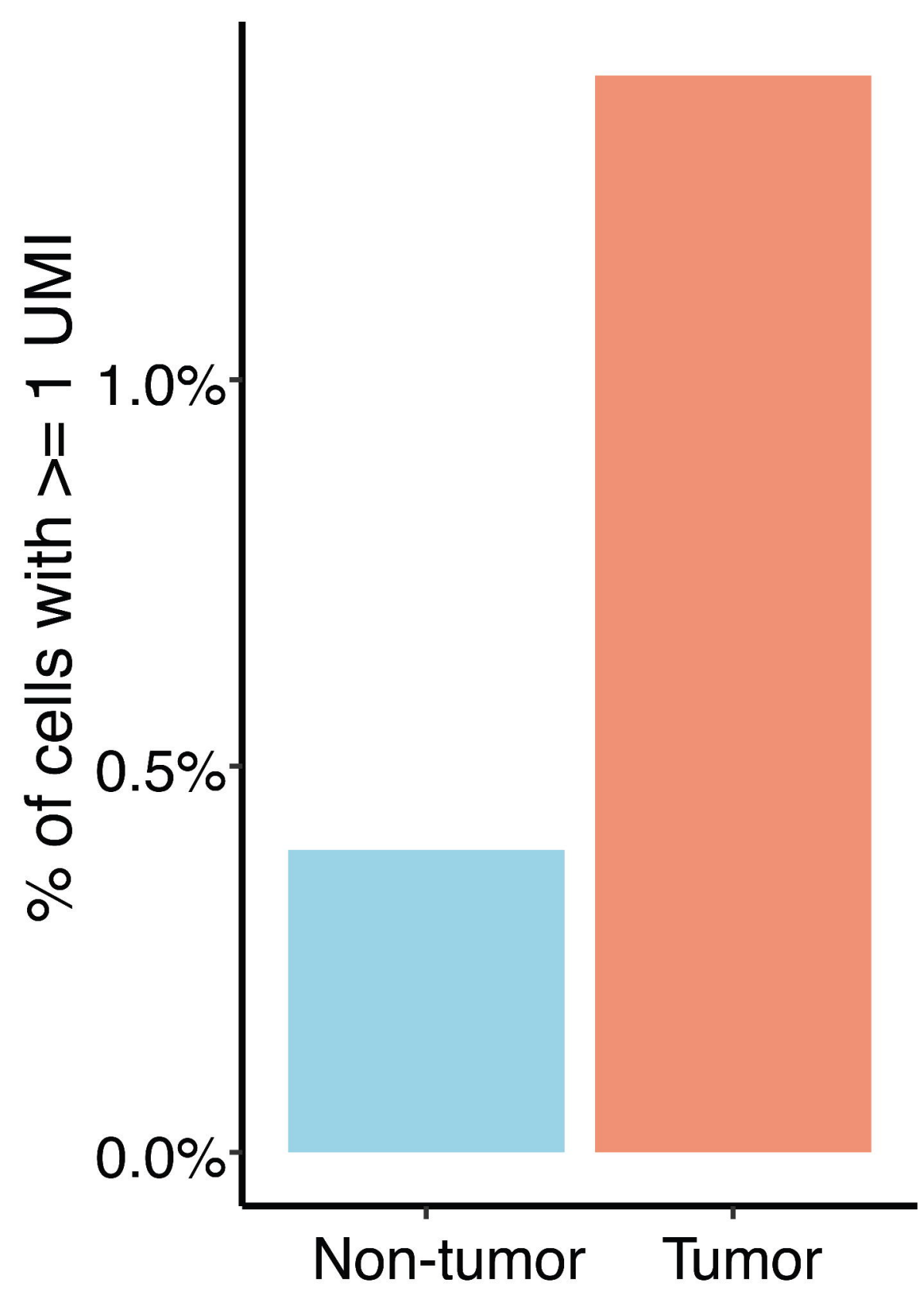


A Differentially expressed genes in infected tumors

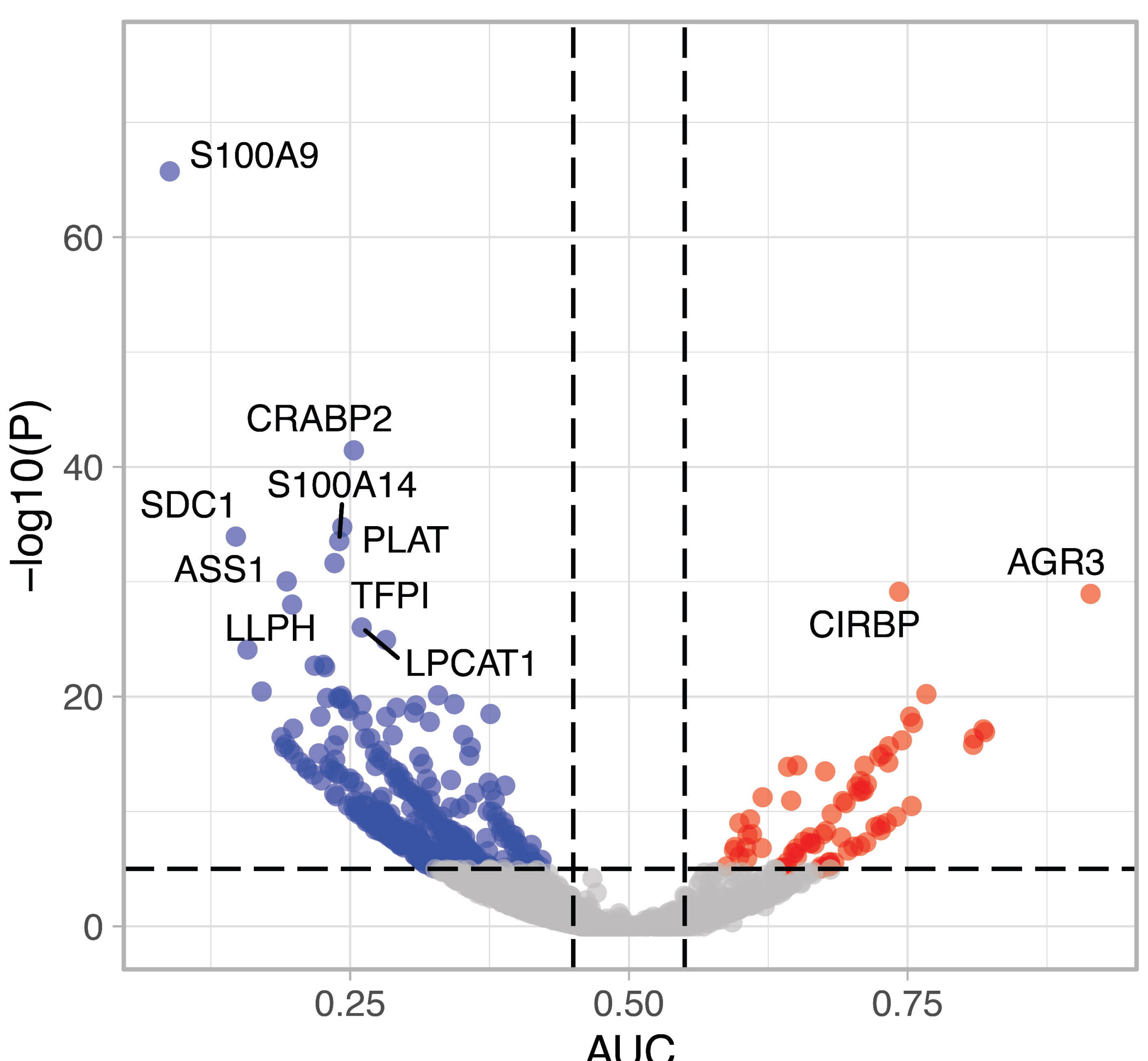

B Gene sets enriched in infected tumors

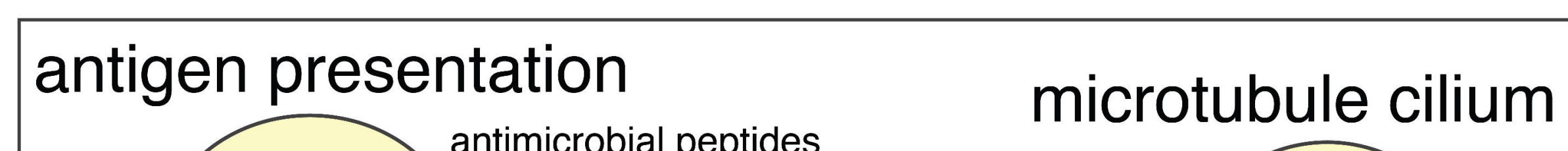

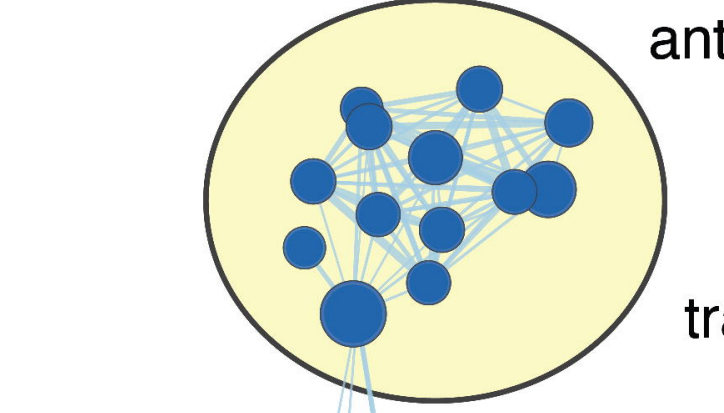

chemotaxis

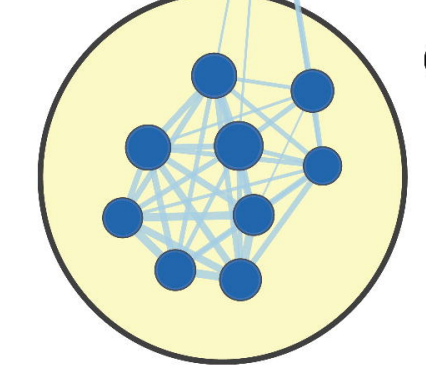

cell killing

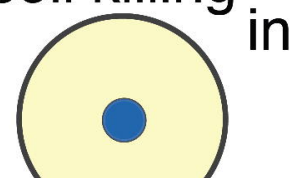

ate response
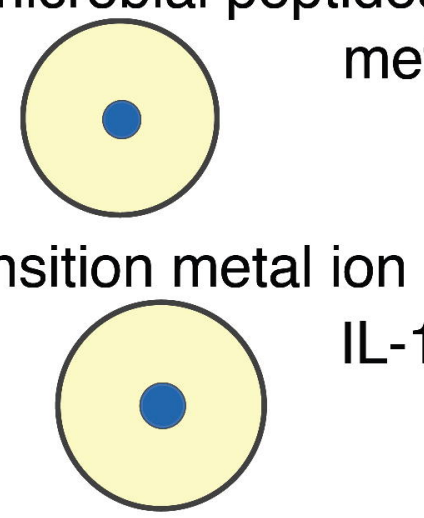

IL-12 production
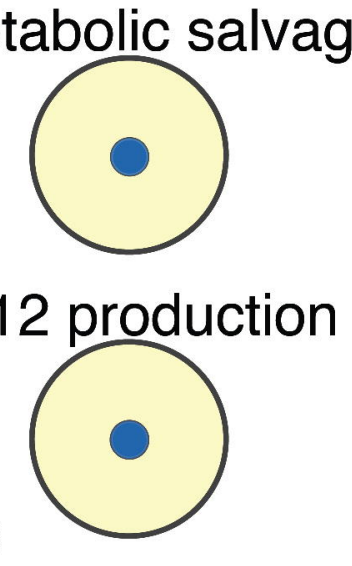

(o) type 1 IFN response IFN-gamma response

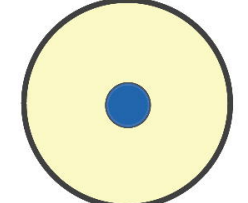

peptidase activity
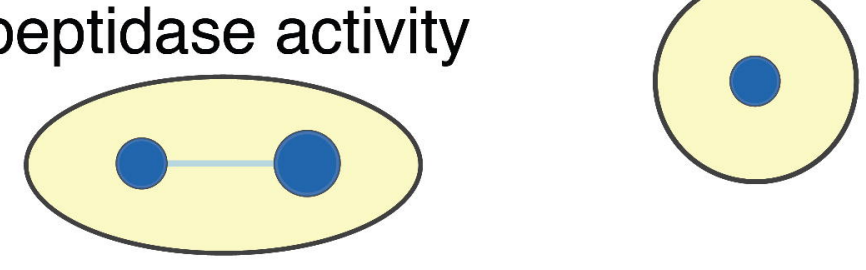

IFN-beta response

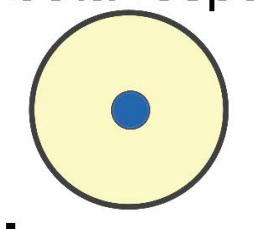

electron respiratory chain

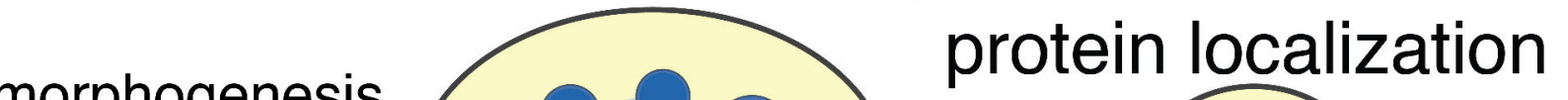

morphogenesis
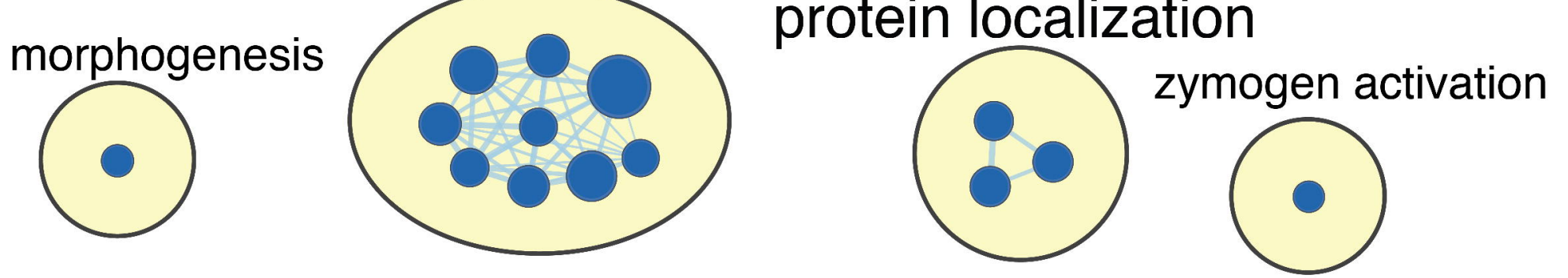

Node

Edge

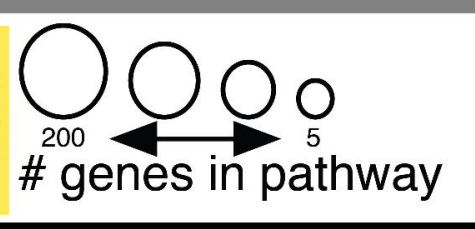

흥

down-regulated in infected tumors -regulated in

up-regulated in

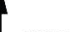

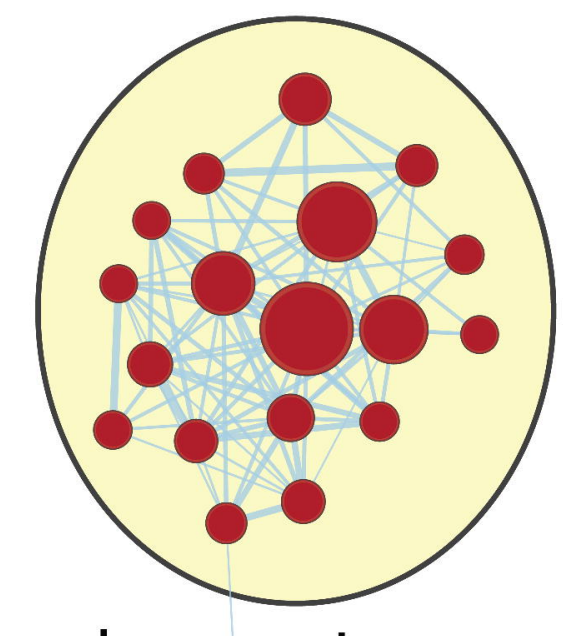

male gamete germ

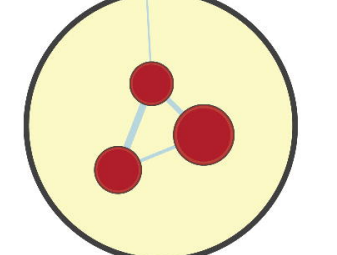

cold response

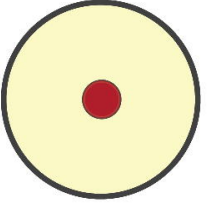

AUC

overlap 


\section{Salmonella}

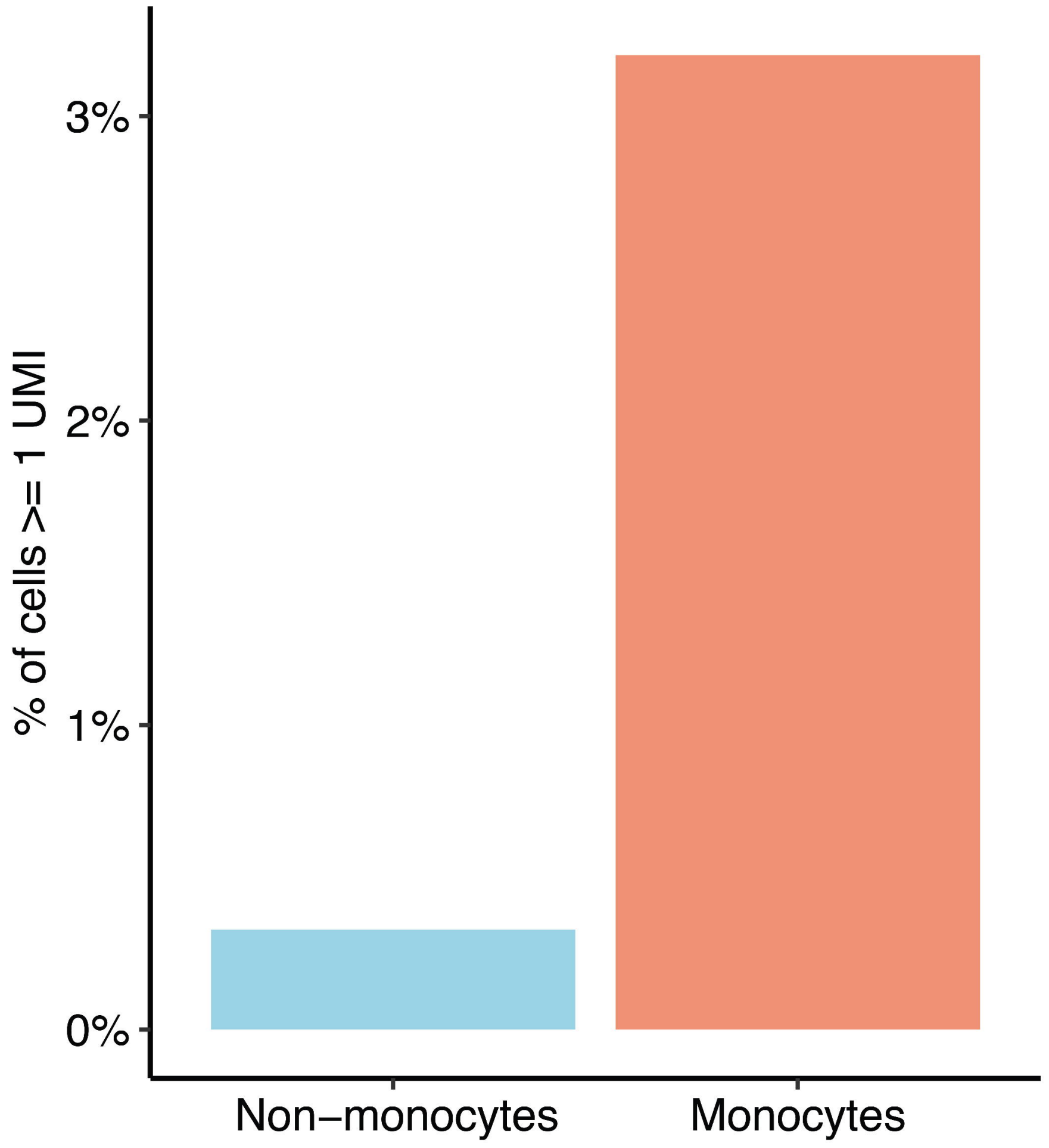


Human

polyomavirus 5

Alphapolyomavirus

Polyomaviridae

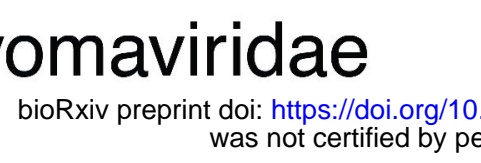

Sepolyvirales

Papovaviricetes

9245-3 tumor versus non-tumor cells

1

Odds ratio (conditional MLE / $95 \% \mathrm{Cl}$ )

B

Human

polyomavirus 5

Alphapolyomavirus

Polyomaviridae

Sepolyvirales

Papovaviricetes
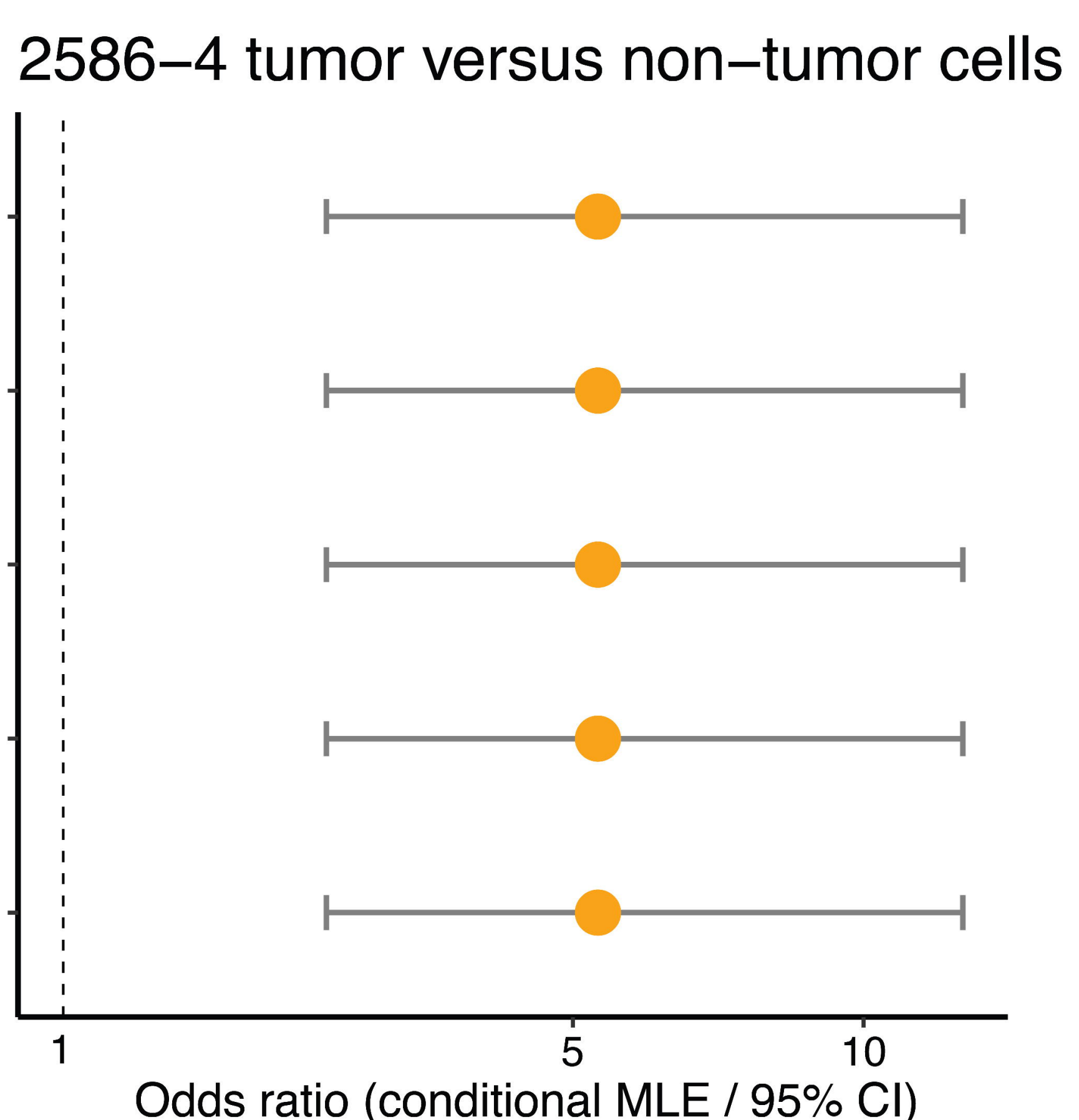

Taxonomy

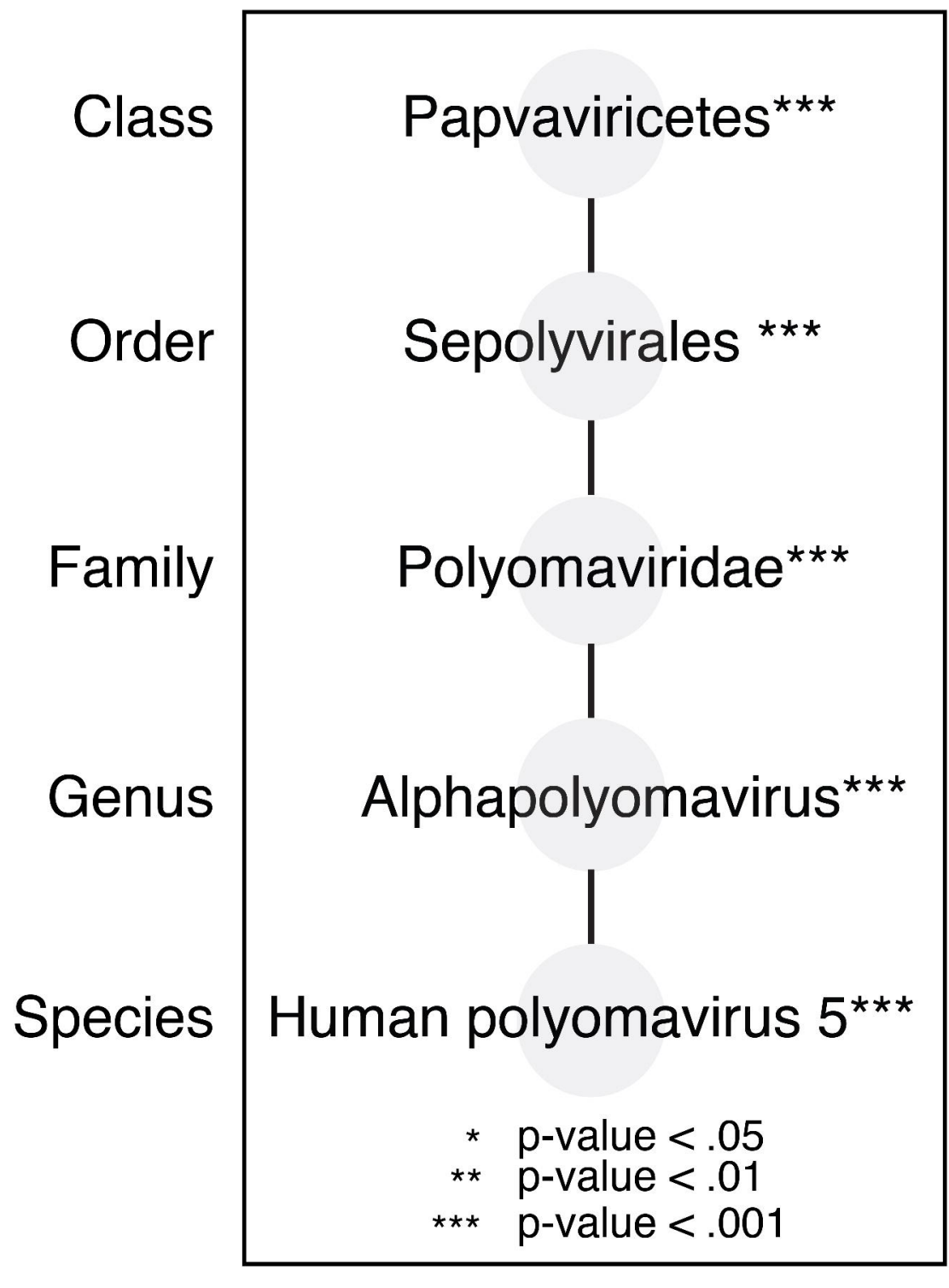

Taxonomy

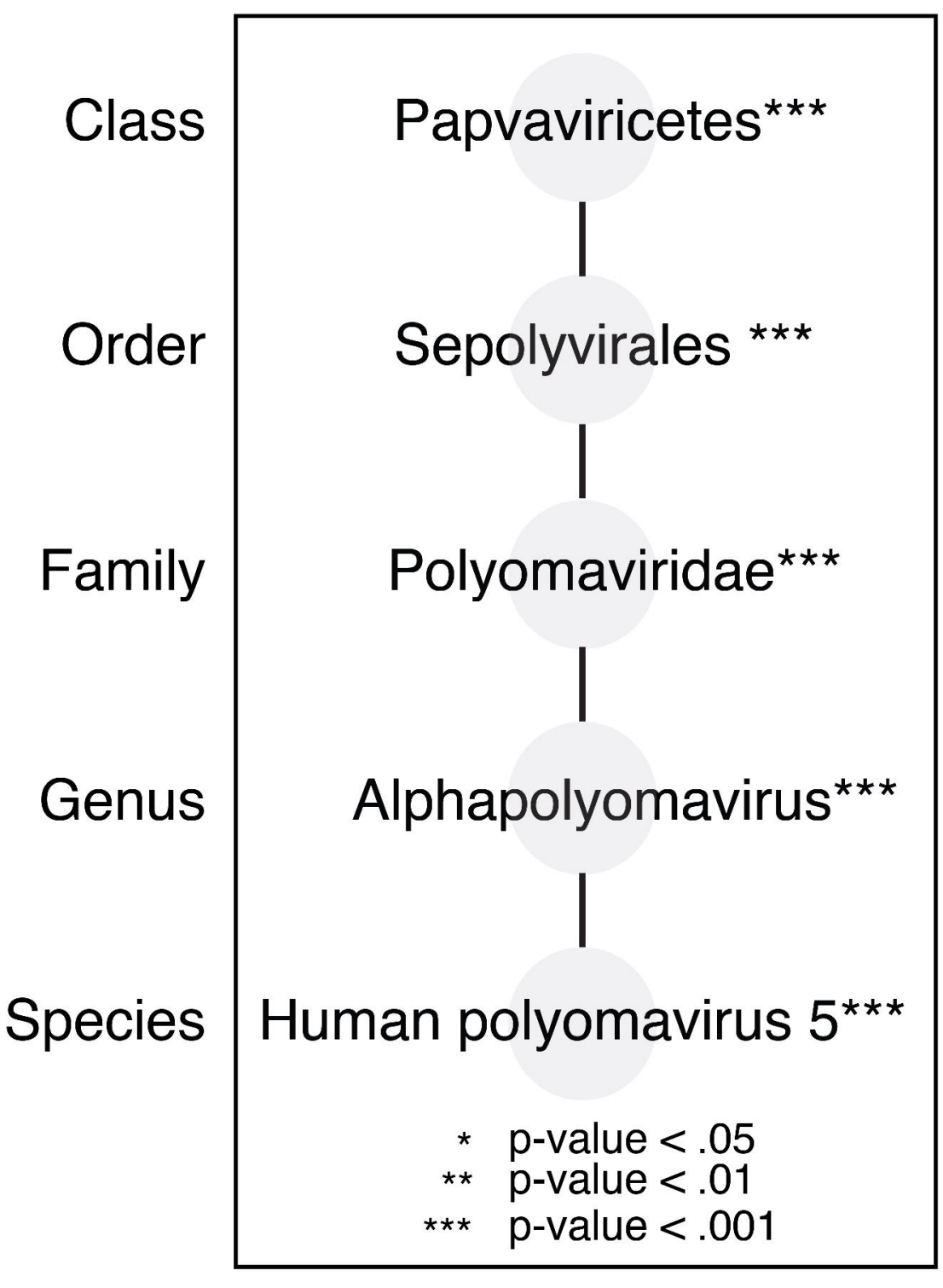

Human polyomavirus 5

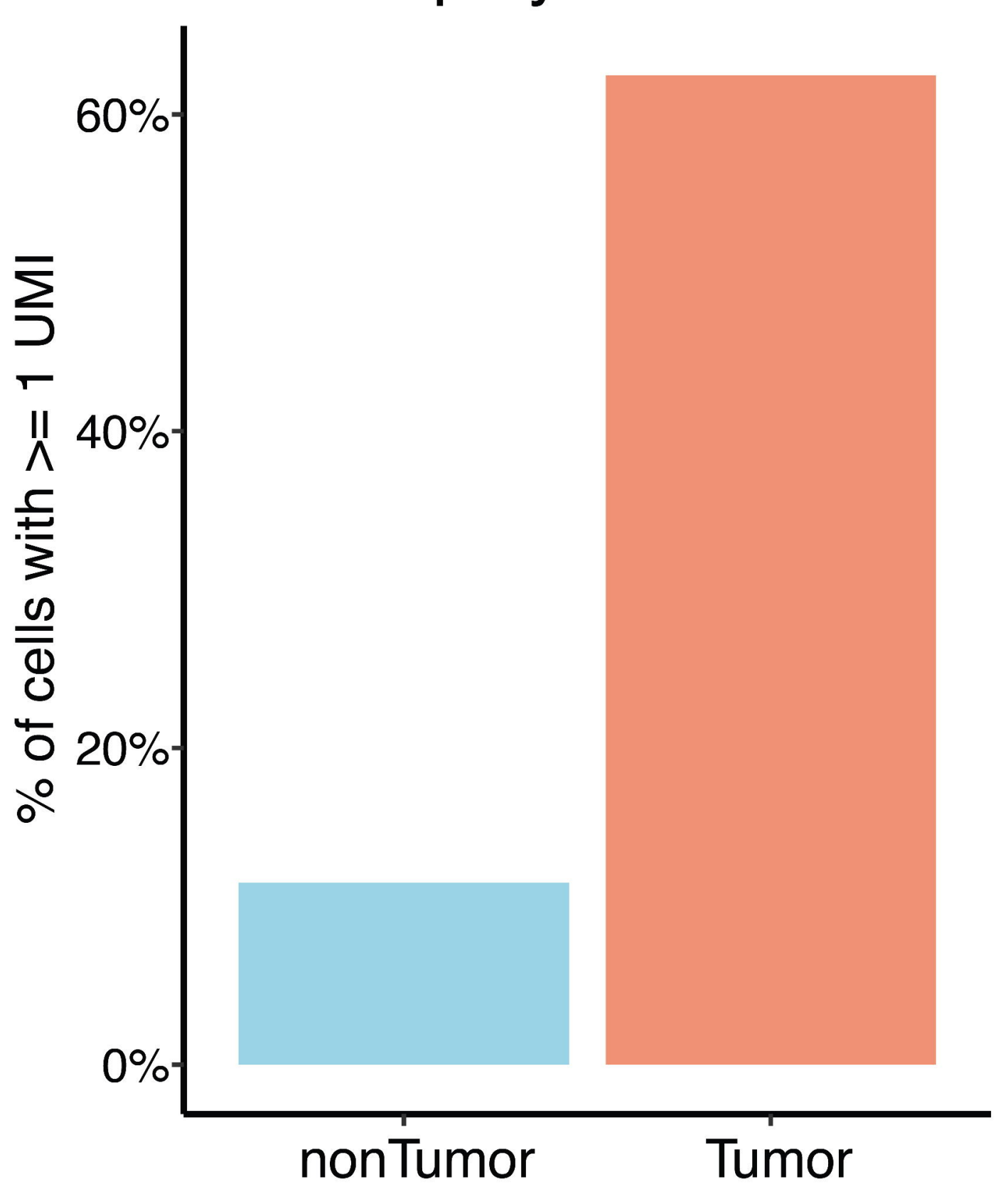

Human polyomavirus 5

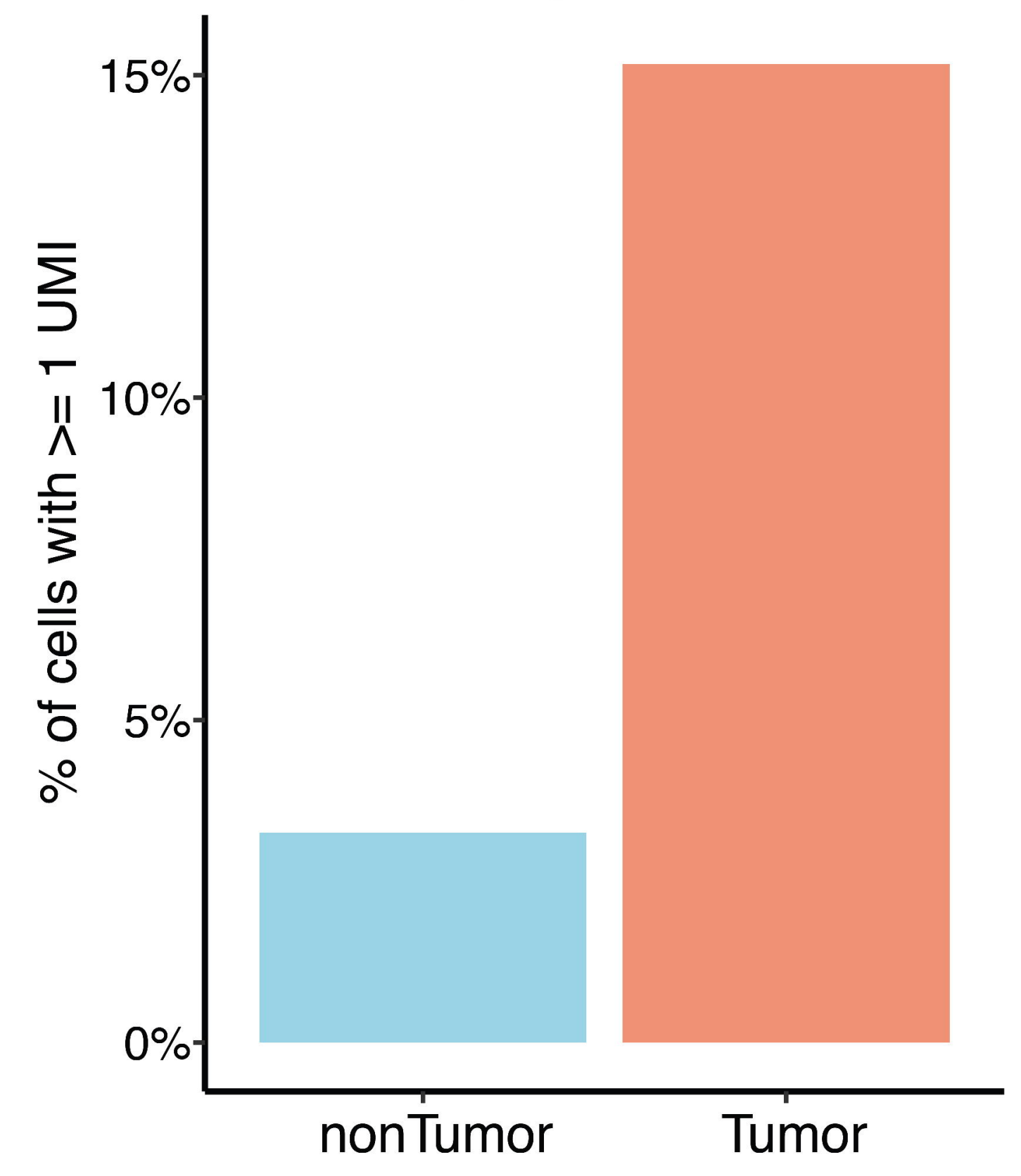

